ESAIM: PROCEEDINGS, June 2008, Vol. 23, p. 10-29

L. Boudin, C. Grandmont, Y. Maday, B. Maury, B. Sapoval \& J.-F. Gerbeau, Editors

\title{
MULTISCALE MODELLING OF THE RESPIRATORY TRACK: A THEORETICAL FRAMEWORK*
}

\author{
C. Grandmont ${ }^{1}$, B. Maury ${ }^{2}$ and A. Sounlah ${ }^{3}$
}

\begin{abstract}
We propose here a decomposition of the respiratory tree into three stages which correspond to different mechanical models. The resulting system is described by the Navier-Stokes equation coupled with an ODE (a simple spring model) representing the motion of the diaphragm muscl. We prove that this problem has at least one solution locally in time for any data and, in the special case where the external forces are equal to zero and if the initial conditions are small enough, that the solution exists globally in time. Note moreover that, in the case where the spring stiffness is equal to zero, we obtain an existence result globally in time provided, once again, that the data are small enough. The behaviour of the global model is illustrated by two-dimensional simulations.
\end{abstract}

Résumé. Afin de décrire l'écoulement de l'air dans les voies aériennes supérieures et proximales, nous proposons un modéle multiéchelles basé sur la décomposition en trois régions de l'arbre trachéobronchique. Dans chacune de ces régions les comportements mécaniques sont différents. Les équations de Navier-Stokes décrivent l'écoulement de l'air dans la partie supérieure et elles sont couplées à une équation différentielle ordinaire modélisant le mouvement du diaphragme et du parenchyme pulmonaire. Pour ce systeme couplé nous démontrons l'existence de solutions faibles en temps petit pour données quelconques, ainsi que l'existence globale en temps dans le cas, très particulier, où la donnée initiale est suffisamment petite et où aucune force extérieure n'est appliquée au système. De plus, si la raideur du ressort modélisant l'élasticité du poumon est nulle, nous obtenons un résultat d'existence globale pour des données petites (conditions initiales et forces appliquées. Nous illustrons le comportement du modèle par des simulations numériques bi-dimensionnelles.

\section{INTRODUCTION, MODELLING ASPECTS}

Breathing involves gas transport through the respiratory track with its visible ends, nose and mouth. Air then streams from the pharynx down to the trachea. The trachea extends from the neck into the thorax, where it divides into right and left main bronchi, which enter the corresponding lungs. The inhaled air is then convected in the bronchus tree which ends in the alveoli embedded in a viscoelastic tissue, made in particular of blood capillaries, and where gaseous exchange occurs. Each lung is enclosed in a space bounded below by the diaphragm and laterally by the chest wall. The air movement is achieved by the displacement of the diaphragm and parenchyma tissue. But the lung may fail to maintain an adequate supply of air. Accidental inhalation of liquid or solid, asthma crisis, pathologies changing the elastic behaviour of the parenchyma (like emphysema or fibrosis), obstructed nose, cancer may occur. The lung is then a place where many exchanges and interactions

\footnotetext{
* This work has been supported by the ACINIM project Lepoumonvousdisje and the french embassy in Tunisia.

1 INRIA, Projet REO Rocquencourt, BP 10578153 Le Chesnay Cedex, France

${ }^{2}$ Laboratoire de mathmatiques, Université Paris-Sud Bâtiment 425, bureau 130, 91405 Orsay Cedex, France

3 Laboratoire de mathématiques , Université Paris-Sud Bâtiment 425, bureau 130, 91405 Orsay Cedex, France
} 
take place, and a numerical lung would certainly be very helpful in the understanding of some of the deseases and a way to guide the intuition for curative gestures. At the time being, the complex fractal geometry of the airway tree makes the air flow simulation on the whole tree unreachable. Note, moreover, that the distal airways from generation 7 cannot be visualised by common medical imaging techniques. Consequently, it is necessary to find new efficient strategies, including simple but realistic models. One possible choice is to try to describe the evolution of the air flux by a simple ODE as it is done in [11]. But even if the model can give valuable hints to understand the respiration mechanisms it can not provide precise informations on the full $3 D$ flow. Our aim is to obtain a model that describes accurately the air flow in the proximal part. To achieve this, one can not forget that this flow is dependent of the distal part and driven by the motion of the diaphragm and parenchyma. One solution will be to find physiological boundary conditions. Yet no such "in vivo" pressure or velocity measurement is available. Thus our aim is to obtain a simplified description of the distal part and what we propose is a decomposition of the respiratory tree into three stages where different models will be exploited and in which the mechanical behaviour is quite different:

- the upper part (up to the 6th generation), where the Navier-Stokes equations hold to describe the fluid flow,

- the distal part (from the 7th to the 17th generation), where one can assume that the Poiseuille law is satisfied in each bronchiole,

- the acini, where the oxygen diffusion takes place and which are embedded in an elastic medium, the parenchyma.

We will assume that the pressure is uniform in the acini part and that they are embedded in a box representing the parenchyma. The motion of the diaphragm and the parenchyma is described by a simple spring model. The decomposition can be schematized by the following figure:

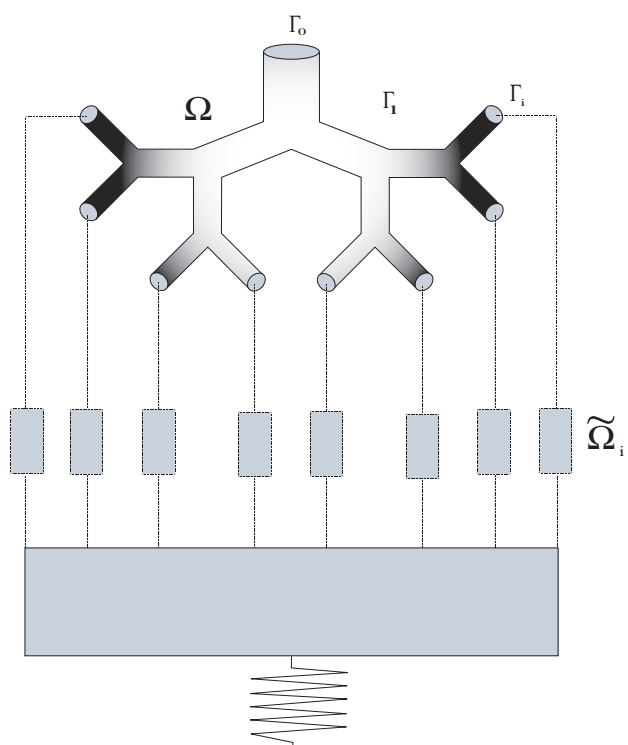

Figure 1. Multiscale model

The "outlet" $\Gamma_{i}, 1 \leq i \leq N$ of the upper part are coupled with Poiseuille flows themselves coupled with the spring motion. We will assume that the velocity profile on the "outlets" $\Gamma_{i}, 1 \leq i \leq N$ is given and, for instance, is a parabolic profile. The assumption is reasonnable since we assume that the Poiseuille law holds true from the 7 th to the 17 th generation of the bronchial tree. Note that the same type of multiscale modelling has been investigated for different applications such as blood flow simulations ( [15], [19]) or air flow simulation ( [8]).

The paper is organized as follows: in a first part we present the coupled system and its variational formulation then we derive, at least formally, a priori error estimates. The main difficulty is to estimate the nonlinear terms 
that is to say the flux at the artificial boundaries $\Gamma_{i}$ of system kinetic energy. In a second part we prove, thanks to a Galerkin approach, that there exists at least one solution, locally in time. The proof is based on standard arguments: energy estimates, obtention of additional bounds in order to pass to the limit in the nonlinear convection terms. Finally we give $2 D$ numerical evidence that this model can reproduce some aspects of normal or pathological breathing.

\section{Problem Setting}

In the upper part, denoted by $\Omega$ we assume that the Navier-Stokes equations hold:

$$
\left\{\begin{aligned}
\rho \frac{\partial \mathbf{u}}{\partial t}+\rho(\mathbf{u} \cdot \nabla) \mathbf{u}-\mu \Delta \mathbf{u}+\nabla p & =0 & & \text { in } \Omega, \\
\nabla \cdot \mathbf{u} & =0 & & \text { in } \Omega, \\
\mathbf{u} & =0 & & \text { on } \Gamma_{\ell}, \\
\mathbf{u} & =\lambda_{i} \mathbf{U}_{i} & & \text { on } \Gamma_{i}, i=0, \ldots, N, \\
\int_{\Gamma_{0}}(\mu \nabla \mathbf{u} \cdot \mathbf{n}-p \mathbf{n}) \cdot \mathbf{U}_{0} & =-P_{0} \int_{\Gamma_{0}} \mathbf{U}_{0} \cdot \mathbf{n} & & \text { on } \Gamma_{0}, \\
\int_{\Gamma_{i}}(\mu \nabla \mathbf{u} \cdot \mathbf{n}-p \mathbf{n}) \cdot \mathbf{U}_{i} & =-\Pi_{i} \int_{\Gamma_{i}} \mathbf{U}_{i} \cdot \mathbf{n} & & \text { on } \Gamma_{i} \quad i=1, \ldots, N,
\end{aligned}\right.
$$

where $\mathbf{u}$ and $p$ are respectivelly the fluid velocity and the fluid pressure. On the lateral trachea boundary $\Gamma_{\ell}$ we impose non-slip boundary conditions on the velocity, whereas on the artificial boundary $\Gamma_{i}, 0 \leq i \leq N$ we consider two types of boundary conditions. Firstly we assume that, on each outlet, the trace of the fluid velocity is proportional to a given velocity profile $\mathbf{U}_{i}$. The proportionality coefficient $\lambda_{i}$ mesures the velocity flux at the interface and is an unknown of the problem. The second type of boundary condition corresponds to a pressure force exerted on the boundary. Note that the first type of boundary condition means that the velocity is reduced to one degree of freedom on the outlets $\Gamma_{i}, 0 \leq i \leq N$, as a consequence, the second type of boundary condition is imposed in a weak form (in some sense a mean value for the normal stress tensor is prescribed). The pressure $P_{0}$ is given whereas the pressures $\Pi_{i}$ are unknown that depend on the dowstream parts. Each of the $\tilde{\Omega}_{i}$ should be a dyadic tree in which we assume that the flow is laminar. Thus, proceeding as in [12] and [9] and by analogy with an electric network, we can consider that the flow is caracterized by a unique equivalent resistance that depends on each resistance of the local branches. Thus, each of the $\tilde{\Omega}_{i}$ is considered as a cylindrical domain, where the flow satisfies a Poiseuille law:

$$
\Pi_{i}-P_{i}=R_{i} \int_{\Gamma_{i}} \mathbf{u} \cdot \mathbf{n}, R_{i} \geq 0,
$$

where $R_{i}$ denotes the equivalent resistance of the distal tree and $P_{i}$ is an alveola pressure. Note that $R_{i}$ depends on the geometric properties (length and diameter) of all the branches of the $i$-th subtree. Moreover, we assume that all the alveola pressures are equal: $P_{i}=P$. Finally, we suppose that these alveoli are embedded in a box filled with an incompressible medium that represents the parenchyma. One part of the box is connected to a spring that governs the diaphragm and parenechyma motion. The equation satisfied by the position $x$ of the diaphragm writes:

$$
m \ddot{x}=-k x+f_{\text {ext }}+f_{P},
$$

where $m$ is the total mass of the lung, $k$ is the stiffness of the spring (that characterises the elastic behavior of the lung) and $f_{\text {ext }}$ is the force developped by the diaphragm during inspiration and forced expiration. In order to couple this simple ODE to the upper part of the model, we have to define $f_{P}$ that stands for the pressure 
force applied by the flow on the elastic medium. If we denote by $S$ the surface of the moving boundary box (diaphragm surface), we have

$$
f_{P}=P S .
$$

Moreover, since the flow is incompressible and since we assume that the parenchyma is made of an incompressible medium, the flow volume variation is equal to the volume variation of the parenchyma box, thus we have

$$
S \dot{x}=\sum_{i=1}^{N} \int_{\Gamma_{i}} \mathbf{u} \cdot \mathbf{n}=-\int_{\Gamma_{0}} \mathbf{u} \cdot \mathbf{n}=-\lambda_{0} \int_{\Gamma_{0}} \mathbf{U}_{0} \cdot \mathbf{n} .
$$

Thus the coupled problem can be written as follows:

$$
\left\{\begin{array}{rlrl}
\rho \frac{\partial \mathbf{u}}{\partial t}+\rho(\mathbf{u} \cdot \nabla) \mathbf{u}-\mu \Delta \mathbf{u}+\nabla p & =0, & & \text { in }(0, T) \times \Omega, \\
\nabla \cdot \mathbf{u} & =0, & \text { in }(0, T) \times \Omega, \\
\mathbf{u} & =0, & \text { on }(0, T) \times \Gamma_{\ell}, \\
\mathbf{u} & =\lambda_{i} \mathbf{U}_{i}, & \text { on }(0, T) \times \Gamma_{i}, \\
& i=0, \ldots, N, \\
\int_{\Gamma_{0}}(\mu \nabla \mathbf{u} \cdot \mathbf{n}-p \mathbf{n}) \cdot \mathbf{U}_{0} & =-P_{0} \int_{\Gamma_{0}} \mathbf{U}_{0} \cdot \mathbf{n}, & & \text { on }(0, T) \times \Gamma_{0}, \\
\int_{\Gamma_{i}}(\mu \nabla \mathbf{u} \cdot \mathbf{n}-p \mathbf{n}) \cdot \mathbf{U}_{i} & =-P \int_{\Gamma_{i}} \mathbf{U}_{i} \cdot \mathbf{n}-R_{i} \lambda_{i}\left(\int_{\Gamma_{i}} \mathbf{U}_{i} \cdot \mathbf{n}\right)^{2}, & & \text { on }(0, T) \times \Gamma_{i}, \\
m \ddot{x}+k x & =f_{e x t}+S P, & & \\
S \dot{x} & =-\lambda_{0} \int_{\Gamma_{0}} \mathbf{U}_{0} \cdot \mathbf{n} . &
\end{array}\right.
$$

This system of equations have to be completed by initial conditions

$$
\left.(\mathbf{u}, x, \dot{x})\right|_{t=0}=\left(\mathbf{u}_{0}, x_{0}, x_{1}\right), \text { with } S x_{1}=-\int_{\Gamma_{0}} \mathbf{u}_{0} \cdot \mathbf{n} .
$$

One particularity of this system is that all the outlets $\Gamma_{i}, 1 \leq i \leq N$ are coupled. This is not the case, for instance, in [15] or [19] where the same type of multiscale modelling is performed but for blood flow simulations. Note that the elastic behavior of the lung is described by only one degree of freedom. Moreover, in the whole coupled model we have only few parameters to fit: $m, k, S, f_{\text {ext }}$ and the resistances $R_{i}$. In particular by modifying $k$ and $R_{i}$ one could obtain pathological behaviors such as asthma (increase of the resistances) or emphysema (decrease of $k$ ). Nevertheless the considered spring model is a very simple one and some aspects of the respiratory cycle can not be reproduced by such a simple model. We refer to [11] for a more complete spring model.

\subsection{A priori estimates}

We start to derive, at least formally, a priori estimates for the coupled system. We multiply the Navier-Stokes equation by $\mathbf{u}$ and integrate over $\Omega$, by taking into account the profile constraint $\mathbf{u}=\lambda_{i} \mathbf{U}_{i}$ on $\Gamma_{i}$ :

$$
\begin{aligned}
& \frac{\rho}{2} \frac{d}{d t} \int_{\Omega}|\mathbf{u}|^{2}+\rho \int_{\Omega}(\mathbf{u} \cdot \nabla \mathbf{u}) \mathbf{u}+\mu \int_{\Omega}|\nabla \mathbf{u}|^{2}+\sum_{i=1}^{N} R_{i}\left(\lambda_{i}\right)^{2}\left(\int_{\Gamma_{i}} \mathbf{U}_{i} \cdot \mathbf{n}\right)^{2}+P_{0} \lambda_{0}\left(\int_{\Gamma_{0}} \mathbf{U}_{0} \cdot \mathbf{n}\right) \\
& +P\left(\sum_{i=1}^{N} \lambda_{i} \int_{\Gamma_{i}} \mathbf{U}_{i} \cdot \mathbf{n}\right)=0,
\end{aligned}
$$


which can be written, by using the fluid incompressibility

$$
\begin{aligned}
& \frac{\rho}{2} \frac{d}{d t} \int_{\Omega}|\mathbf{u}|^{2}+\rho \int_{\Omega}(\mathbf{u} \cdot \nabla \mathbf{u}) \mathbf{u}+\mu \int_{\Omega}|\nabla \mathbf{u}|^{2}+\sum_{i=1}^{N} R_{i}\left(\lambda_{i}\right)^{2}\left(\int_{\Gamma_{i}} \mathbf{U}_{i} \cdot \mathbf{n}\right)^{2}+P_{0} \lambda_{0}\left(\int_{\Gamma_{0}} \mathbf{U}_{0} \cdot \mathbf{n}\right) \\
& -P \lambda_{0}\left(\int_{\Gamma_{0}} \mathbf{U}_{0} \cdot \mathbf{n}\right)=0 .
\end{aligned}
$$

Moreover we can write the convective terms, thanks to an integration by parts, as a flux of kinetic energy at the inlet and at the outlets:

$$
\begin{aligned}
& \frac{\rho}{2} \frac{d}{d t} \int_{\Omega}|\mathbf{u}|^{2}+\frac{\rho}{2} \sum_{i=0}^{N} \int_{\Gamma_{i}}|\mathbf{u}|^{2}(\mathbf{u} \cdot \mathbf{n})+\mu \int_{\Omega}|\nabla \mathbf{u}|^{2}+\sum_{i=1}^{N} R_{i}\left(\lambda_{i}\right)^{2}\left(\int_{\Gamma_{i}} \mathbf{U}_{i} \cdot \mathbf{n}\right)^{2}+P_{0} \lambda_{0}\left(\int_{\Gamma_{0}} \mathbf{U}_{0} \cdot \mathbf{n}\right) \\
& -P \lambda_{0}\left(\int_{\Gamma_{0}} \mathbf{U}_{0} \cdot \mathbf{n}\right)=0 .
\end{aligned}
$$

Then we multiply the spring equation by $\dot{x}$

$$
\frac{m}{2} \frac{d}{d t}|\dot{x}|^{2}+\frac{k}{2} \frac{d}{d t}|x|^{2}=f_{\text {ext }} \dot{x}+P S \dot{x} .
$$

Now using the fact that $S \dot{x}=-\lambda_{0}\left(\int_{\Gamma_{0}} \mathbf{U}_{0} \cdot \mathbf{n}\right)$ and adding (8), (9) we obtain

$$
\begin{aligned}
& \frac{\rho}{2} \frac{d}{d t} \int_{\Omega}|\mathbf{u}|^{2}+\frac{\rho}{2} \sum_{i=0}^{N} \int_{\Gamma_{i}}|\mathbf{u}|^{2}(\mathbf{u} \cdot \mathbf{n})+\mu \int_{\Omega}|\nabla \mathbf{u}|^{2}+\sum_{i=1}^{N} R_{i}\left(\lambda_{i}\right)^{2}\left(\int_{\Gamma_{i}} \mathbf{U}_{i} \cdot \mathbf{n}\right)^{2} \\
& +\frac{m}{2} \frac{d}{d t}|\dot{x}|^{2}+\frac{k}{2} \frac{d}{d t}|x|^{2}=P_{0} S \dot{x}+f_{\text {ext }} \dot{x} .
\end{aligned}
$$

Identity (10) represents the energy balance of the coupled system. In particular, the energy is dissipated: as for the standard Navier-Stokes equations the fact that the flow is viscous contribute to the dissipation of the energy but here there is a second contribution to the dissipation that comes from the resistive part of the bronchial tree, namely

$$
\sum_{i=1}^{N} R_{i}\left(\lambda_{i}\right)^{2}\left(\int_{\Gamma_{i}} \mathbf{U}_{i} \cdot \mathbf{n}\right)^{2}=\sum_{i=1}^{N} R_{i}\left(\int_{\Gamma_{i}} \mathbf{u} \cdot \mathbf{n}\right)^{2} .
$$

In order to obtain energy estimates we have to estimate this flux of kinetic energy at the interface, that writes, taking into account the profile constraint at the interface $\left(\mathbf{u}=\lambda_{i} \mathbf{U}_{i}\right.$ on $\left.\Gamma_{i}\right)$

$$
\sum_{i=0}^{N} \int_{\Gamma_{i}}|\mathbf{u}|^{2}(\mathbf{u} \cdot \mathbf{n})=\sum_{i=0}^{N}\left(\lambda_{i}\right)^{3} \int_{\Gamma_{i}}\left|\mathbf{U}_{i}\right|^{2}\left(\mathbf{U}_{i} \cdot \mathbf{n}\right) .
$$

First of all we introduce the following functional spaces (we recall that $\Gamma_{\ell}$ is the lateral boundary):

$$
\begin{gathered}
H=\left\{\mathbf{v} \in L^{2}(\Omega), \nabla \cdot \mathbf{v}=0, \mathbf{v} \cdot \mathbf{n}=0 \text { on } \Gamma_{\ell}\right\} \\
V=\left\{\mathbf{v} \in H^{1}(\Omega), \nabla \cdot \mathbf{v}=0, \mathbf{v}=0 \text { on } \Gamma_{\ell}\right\} \\
W=\left\{\mathbf{v} \in V, \exists \tilde{\Lambda}=\left(\tilde{\lambda}_{i}\right)_{0 \leq i \leq N} \text { s. t. } \mathbf{v}=\tilde{\lambda}_{i} \mathbf{U}_{i} \text { on } \Gamma_{i}, \quad i=0, \ldots, N\right\} .
\end{gathered}
$$

To bound the nonlinear convection term we will use the following lemma: 
Lemma 1.1. If $\mathbf{U}_{i} \cdot \mathbf{n} \neq 0$ and if $\mathbf{U}_{i} \cdot \mathbf{n} \in\left(H_{00}^{\frac{1}{2}}\left(\Gamma_{i}\right)\right)^{\prime}$ there exists a constant $C_{i}>0$ such that, for all $\mathbf{v} \in W$, we have

$$
\left|\tilde{\lambda}_{i}\right| \leq C_{i}\|\mathbf{v}\|_{L^{2}(\Omega)}, \quad i=0, \ldots, N
$$

Proof. It is an easy task to verify, using standard arguments (see for instance [7]) that

$$
\|\mathbf{v} \cdot \mathbf{n}\|_{\left(H_{00}^{1 / 2}\left(\Gamma_{i}\right)\right)^{\prime}} \leq C\left(\|\mathbf{v}\|_{L^{2}(\Omega)}+\|\nabla \cdot \mathbf{v}\|_{L^{2}(\Omega)}\right), \forall \mathbf{v} \in H_{\text {div }}(\Omega)=\left\{\mathbf{v} \in L^{2}(\Omega) ; \nabla \cdot \mathbf{v} \in L^{2}(\Omega)\right\}
$$

Here the space $H_{00}^{1 / 2}\left(\Gamma_{i}\right)$ denotes the space of trace function that, if extended by zero over $\partial \Omega$, belongs to $H^{1 / 2}(\partial \Omega)$. But for all $\mathbf{v} \in W$ we have

$$
\left|\tilde{\lambda}_{i}\right|=\frac{\|\mathbf{v} \cdot \mathbf{n}\|_{\left(H_{00}^{1 / 2}\left(\Gamma_{i}\right)\right)^{\prime}}}{\left\|\mathbf{U}_{i} \cdot \mathbf{n}\right\|_{\left(H_{00}^{1 / 2}\left(\Gamma_{i}\right)\right)^{\prime}}} .
$$

Then taking into account the fact that $\nabla \cdot \mathbf{v}=0$, we have

$$
\left|\tilde{\lambda}_{i}\right| \leq \tilde{C}_{i}\|\mathbf{v}\|_{L^{2}(\Omega)} \text { where } \tilde{C}_{i}=\frac{C}{\left\|\mathbf{U}_{i} \cdot \mathbf{n}\right\|_{\left(H_{00}^{1 / 2}\left(\Gamma_{i}\right)\right)^{\prime}}} .
$$

Lemma 1.1 enables us to estimate the nonlinear convective term

$$
\left.\left|\sum_{i=0}^{N} \int_{\Gamma_{i}}\right| \mathbf{u}\right|^{2}(\mathbf{u} \cdot \mathbf{n})|=| \sum_{i=0}^{N}\left(\lambda_{i}\right)^{3} \int_{\Gamma_{i}}\left|\mathbf{U}_{i}\right|^{2}\left(\mathbf{U}_{i} \cdot \mathbf{n}\right) \mid \leq \Gamma C_{1}\|\mathbf{u}\|_{L^{2}(\Omega)}^{3}
$$

with $\Gamma=\sum_{i=0}^{N} \int_{\Gamma_{i}}\left|\mathbf{U}_{i}\right|^{2} \mid \mathbf{U}_{i} \cdot \mathbf{n}$ and $C_{1}=\max _{0 \leq i \leq N}\left|\tilde{C}_{i}\right|$. Note that this estimate relies on the fact that the trace of the fluid velocity on each $\Gamma_{i}$ is supposed to be described by one parameter. This estimate is, of course, not true in the general case. We will assume, to simplify, that the velocity profils $\mathbf{U}_{i}$ and the resistances $R_{i}$ do not depend on time. Nevertheless the energy estimates derived below hold also if $\left.\mathbf{U}_{i} \in L^{\infty}\left(0, T ; H_{00}^{\frac{1}{2}}\left(\Gamma_{i}\right)\right)\right)$ and $R_{i} \in L^{\infty}(0, T)$.

Using the last estimate and the energy balance (10) yields

$$
\begin{aligned}
& \frac{\rho}{2} \frac{d}{d t} \int_{\Omega}|\mathbf{u}|^{2}+\frac{m}{2} \frac{d}{d t}|\dot{x}|^{2}+\frac{k}{2} \frac{d}{d t}|x|^{2} \\
& +\mu \int_{\Omega}|\nabla \mathbf{u}|^{2}+\sum_{i=1}^{N} R_{i}\left(\lambda_{i}\right)^{2}\left(\int_{\Gamma_{i}} \mathbf{U}_{i} \cdot \mathbf{n}\right)^{2} \\
& \leq\left(\left|S P_{0}\right|+\left|f_{\text {ext }}\right|\right)|\dot{x}|+\frac{\rho}{2} \Gamma C_{1}\|\mathbf{u}\|_{L^{2}(\Omega)}^{3} \\
& \leq C\left(|\dot{x}|^{2}+\|\mathbf{u}\|_{L^{2}(\Omega)}^{3}+\left|S P_{0}\right|^{2}+\left|f_{\text {ext }}\right|^{2}\right) .
\end{aligned}
$$

Thus if $P_{0} \in L^{2}(0, T)$ and $f_{\text {ext }} \in L^{2}(0, T)$, we easily verify that there exist a time $\tau_{0}, 0<\tau_{0}<T$ sufficiently small depending on the data and a constant $C>0$ also depending on the data such that for any regular solution $(\mathbf{u}, x)$ :

$$
\begin{aligned}
& \frac{\rho}{2}\|\mathbf{u}\|_{L^{2}(\Omega)}^{2}(t)+\frac{m}{2}|\dot{x}|^{2}(t)+\frac{k}{2}|x|^{2}(t) \\
& +\mu \int_{0}^{t} \int_{\Omega}|\nabla \mathbf{u}|^{2}+\sum_{i=1}^{N} R_{i} \int_{0}^{t}\left(\lambda_{i}\right)^{2}\left(\int_{\Gamma_{i}} \mathbf{U}_{i} \cdot \mathbf{n}\right)^{2} \leq C, \quad t \leq \tau_{0} .
\end{aligned}
$$


Thus any regular solution $\mathbf{u}$ is bounded by the data in $L^{\infty}\left(0, \tau_{0} ; L^{2}(\Omega)\right) \cap L^{2}\left(0, \tau_{0} ; H^{1}(\Omega)\right)$ that implies in particular that the velocity fluxes and $\dot{x}$ are bounded in $L^{\infty}\left(0, \tau_{0}\right)$.

Note that, due to the bound obtained on the nonlinear convective term, this a priori estimate only holds on a small time interval and will lead to an existence result for large data locally in time.

Nevertheless, if the initial data are small enough and in the special case where no external efforts are applied we can prove that the velocity remains in a given ball for any time. Indeed, using Poincaré inequality,

$$
\|\mathbf{u}\|_{L^{2}(\Omega)} \leq C_{2}\|\nabla \mathbf{u}\|_{L^{2}(\Omega)}
$$

the nonlinear terms can be bounded as follows:

$$
\left.\left|\sum_{i=0}^{N} \int_{\Gamma_{i}}\right| \mathbf{u}\right|^{2}(\mathbf{u} \cdot \mathbf{n}) \mid \leq \Gamma C_{1} C_{2}^{2}\|\mathbf{u}\|_{L^{2}(\Omega)}\|\nabla \mathbf{u}\|_{L^{2}(\Omega)}^{2},
$$

so that

$$
\begin{aligned}
& \frac{\rho}{2} \frac{d}{d t}\|\mathbf{u}\|_{L^{2}(\Omega)}^{2}+\frac{m}{2} \frac{d}{d t}|\dot{x}|^{2}+\frac{k}{2} \frac{d}{d t}|x|^{2}+\left(\frac{\mu}{2}-\frac{K \rho}{2}\|\mathbf{u}\|_{L^{2}(\Omega)}\right)\|\nabla \mathbf{u}\|_{L^{2}(\Omega)}^{2} \\
& +\sum_{i=1}^{N} R_{i}\left(\lambda_{i}\right)^{2}\left(\int_{\Gamma_{i}} \mathbf{U}_{i} \cdot \mathbf{n}\right)^{2} \leq 0 \quad \text { with } K=\Gamma C_{1} C_{2}^{2} .
\end{aligned}
$$

Thus, assuming that $\left\|\mathbf{u}_{0}\right\|_{L^{2}(\Omega)} \leq \frac{\mu}{K \rho}$, we obtain that on any time interval during which $\frac{\mu}{2}-\frac{K \rho}{2}\|\mathbf{u}\|_{L^{2}(\Omega)}>0$

$$
\frac{\rho}{2} \frac{d}{d t}\|\mathbf{u}\|_{L^{2}(\Omega)}^{2}+\frac{m}{2} \frac{d}{d t}|\dot{x}|^{2}+\frac{k}{2} \frac{d}{d t}|x|^{2} \leq 0 .
$$

Moreover, if

$$
\frac{\rho}{2}\left\|\mathbf{u}_{0}\right\|_{L^{2}(\Omega)}^{2}+\frac{m}{2}\left|x_{1}\right|^{2}+\frac{k}{2}\left|x_{0}\right|^{2} \leq \frac{\mu^{2}}{8 \rho K^{2}}
$$

the solution verifies $\frac{\rho}{2}\|\mathbf{u}\|_{L^{2}(\Omega)}^{2}+\frac{m}{2}|\dot{x}|^{2}+\frac{k}{2}|x|^{2} \leq \frac{\mu^{2}}{8 \rho K^{2}}$ for all time.

Remark 1.2. In the special case $P_{0}=0, f_{\text {ext }}=0$ and under the hypothesis $\left\|\mathbf{u}_{0}\right\|_{L^{2}(\Omega)} \leq \mu / \rho K$, the energy of the coupled system is decreasing. Note that this property cannot be expected in general, because an initial velocity with a large $L^{2}$ norm on the inlet is likely to induce, at least transitorily, an increase of global energy by kinetic energy entering the domain. In a similar manner, if the profiles are not prescribed, a highly singular initial velocity on the inlet may produce a similar increase of energy, as small at it may be in $L^{2}$ volumic norm.

One can also obtain estimate globally in time in the special case where $k=0$. Indeed, in this case we have

$$
\begin{aligned}
& \frac{\rho}{2} \frac{d}{d t}\|\mathbf{u}\|_{L^{2}(\Omega)}^{2}+\frac{m}{2} \frac{d}{d t}|\dot{x}|^{2} \\
& +\mu\|\nabla \mathbf{u}\|_{L^{2}(\Omega)}^{2}+\sum_{i=1}^{N} R_{i}\left(\lambda_{i}\right)^{2}\left(\int_{\Gamma_{i}} \mathbf{U}_{i} \cdot \mathbf{n}\right)^{2} \\
& \leq\left(\left|S P_{0}\right|+\left|f_{\text {ext }}\right|\right)|\dot{x}|+\frac{\rho}{2} \Gamma C_{1}\|\mathbf{u}\|_{L^{2}(\Omega)}^{3} .
\end{aligned}
$$

Using Poincaré inequality and since

$$
|\dot{x}|=\left|\frac{\lambda_{0} \int_{\Gamma_{0}} \mathbf{u}_{0} \cdot \mathbf{n}}{S}\right| \leq C\left|\lambda_{0}\right| \leq C_{3}\|\mathbf{u}\|_{L^{2}(\Omega)} \leq C_{3} C_{2}\|\nabla \mathbf{u}\|_{L^{2}(\Omega)},
$$


we obtain

$$
\begin{array}{r}
\frac{\rho}{2} \frac{d}{d t}\|\mathbf{u}\|_{L^{2}(\Omega)}^{2}+\frac{m}{2} \frac{d}{d t}|\dot{x}|^{2}+\left(\frac{\mu}{2}-\frac{K \rho}{2}\|\mathbf{u}\|_{L^{2}(\Omega)}\right)\|\nabla \mathbf{u}\|_{L^{2}(\Omega)}^{2} \\
+\sum_{i=1}^{N} R_{i}\left(\lambda_{i}\right)^{2}\left(\int_{\Gamma_{i}} \mathbf{U}_{i} \cdot \mathbf{n}\right)^{2} \leq \frac{C_{2}^{2} C_{3}^{2}}{2 \mu}\left(\left|S P_{0}\right|^{2}+\left|f_{\text {ext }}\right|^{2}\right)
\end{array}
$$

and using, once again, Poincaré inequality

$$
\begin{gathered}
\frac{\rho}{2} \frac{d}{d t}\|\mathbf{u}\|_{L^{2}(\Omega)}^{2}+\frac{m}{2} \frac{d}{d t}|\dot{x}|^{2}+\frac{1}{C_{2}^{2}}\left(\frac{\mu}{2}-\frac{K \rho}{2}\|\mathbf{u}\|_{L^{2}(\Omega)}\right)\|\mathbf{u}\|_{L^{2}(\Omega)}^{2} \\
+\sum_{i=1}^{N} R_{i}\left(\lambda_{i}\right)^{2}\left(\int_{\Gamma_{i}} \mathbf{U}_{i} \cdot \mathbf{n}\right)^{2} \leq \frac{C_{2}^{2} C_{3}^{2}}{2 \mu}\left(\left|S P_{0}\right|^{2}+\left|f_{\text {ext }}\right|^{2}\right) .
\end{gathered}
$$

Moreover, since $|\dot{x}|^{2} \leq C\|\mathbf{u}\|_{L^{2}(\Omega)}^{2}$ it is easy to obtain an inequality of the type

$$
\frac{d}{d t} \phi+A\left(\frac{\mu}{2}-B \sqrt{\phi}\right) \phi \leq \frac{C}{2 \mu}\left(\left|S P_{0}\right|^{2}+\left|f_{\text {ext }}\right|^{2}\right),
$$

with $\phi=|\dot{x}|^{2}+\|\mathbf{u}\|_{L^{2}(\Omega)}^{2}$. Consequently, assuming that $\sqrt{\phi(0)} \leq \frac{\mu}{4 B}$ and that $\left|S P_{0}\right|^{2}+\left|f_{\text {ext }}\right|^{2} \leq \frac{A \mu^{4}}{32 B^{2} C}$, it is easy to verify that $\sqrt{\phi(t)} \leq \frac{\mu}{4 B}$ for all $t$.

Remark 1.3. Note that the case $k \neq 0$ can not be treated in the same way because of the diaphragm displacement that can not be bounded easily in $L^{\infty}$. Observe, however that from the physical point of view this displacement is bounded (the volume of the chest is given) and one could impose it by considering a more general spring model (see [11]).

Remark 1.4. The previous assumptions on the data involve $\mu$. Note however that one can obtain conditions involving also the resistances $R_{i}$ (provided that the $R_{i}$ are strictly positive and the flux of the velocity profil $\int_{\Gamma_{i}} \mathbf{U}_{i} \cdot \mathbf{n}$ are not equal to zero) by estimating the nonlinear convection term as follows:

$$
\left.\left.\left|\sum_{i=0}^{N} \int_{\Gamma_{i}}\right| \mathbf{u}\right|^{2}(\mathbf{u} \cdot \mathbf{n})\left|\leq C_{3}\|\mathbf{u}\|_{L^{2}(\Omega)} \sum_{i=0}^{N}\right| \lambda_{i}\right|^{2},
$$

and by writting $\lambda_{0}$ (or $\dot{x}$ ) as a linear combinaison of the $\left(\lambda_{i}\right)_{1 \leq i \leq N}$. Yet the resulting conditions are of the same type than the one we just obtained, since the resistances $R_{i}$ are proportional to $\mu$.

\subsection{Variational formulation}

In this subsection we give the variational formulation of the coupled system. But, first we precise the assumptions made on the data

$$
\begin{gathered}
\left(\mathbf{u}_{0}, x_{0}, x_{1}\right) \text { in } H \times \mathbb{R}^{2} \text { with } S x_{1}=-\int_{\Gamma_{0}} \mathbf{u}_{0} \cdot \mathbf{n} \\
P_{0} \in L^{2}(0, T), \quad f_{\text {ext }} \in L^{2}(0, T) \\
R_{i} \geq 0, i=1, \ldots, N \\
\mathbf{U}_{i} \in H_{00}^{\frac{1}{2}}\left(\Gamma_{i}\right), i=0, \ldots, N
\end{gathered}
$$


Let us consider a divergence-free field $\mathbf{v} \in H^{1}(\Omega), \mathbf{v}=0$ on $\Gamma_{\ell},\left.\mathbf{v}\right|_{\Gamma_{i}}=\beta_{i} \mathbf{U}_{i}$, for $0 \leq i \leq N$, and $\phi \in \mathcal{D}([0, t))$. In order to obtain a weak formulation of the coupled system we multiply the Navier-Stokes equations by $\mathbf{v} \phi$ and integrate over $\Omega \times(0, T)$. The spring equation is multiplied by $-\frac{1}{S}\left(\int_{\Gamma_{0}} \mathbf{v} \cdot \mathbf{n}\right) \phi$ and added to the previous contribution. By integrating by parts (in space and in time) the Navier-Stokes equations and by taking into account the constraint $S \dot{x}=-\int_{\Gamma_{0}} \mathbf{u} \cdot \mathbf{n}$ we obtain a weak formulation of the coupled system (6), which leads to the following definition: We shall say that $\mathbf{u}$ is a weak solution of $(6)$ on $[0, T)$ if

- $\mathbf{u} \in L^{2}(0, T ; V) \cap L^{\infty}(0, T ; H)$,

- $\exists \lambda_{i} \in L^{\infty}(0, T)$ such that $\left.\mathbf{u}_{i}\right|_{\Gamma_{i}}=\lambda_{i} \mathbf{U}_{i}$, for a. e. $t$,

- $\forall \mathbf{v} \in H^{1}(\Omega), \nabla \cdot \mathbf{v}=0, \mathbf{v}=0$ on $\Gamma_{\ell}$ and $\exists\left(\tilde{\lambda}_{i}\right)_{0 \leq i \leq N}$ s. t. $\left.\mathbf{v}\right|_{\Gamma_{i}}=\tilde{\lambda}_{i} \mathbf{U}_{i}, \forall \phi \in \mathcal{D}([0, T))$

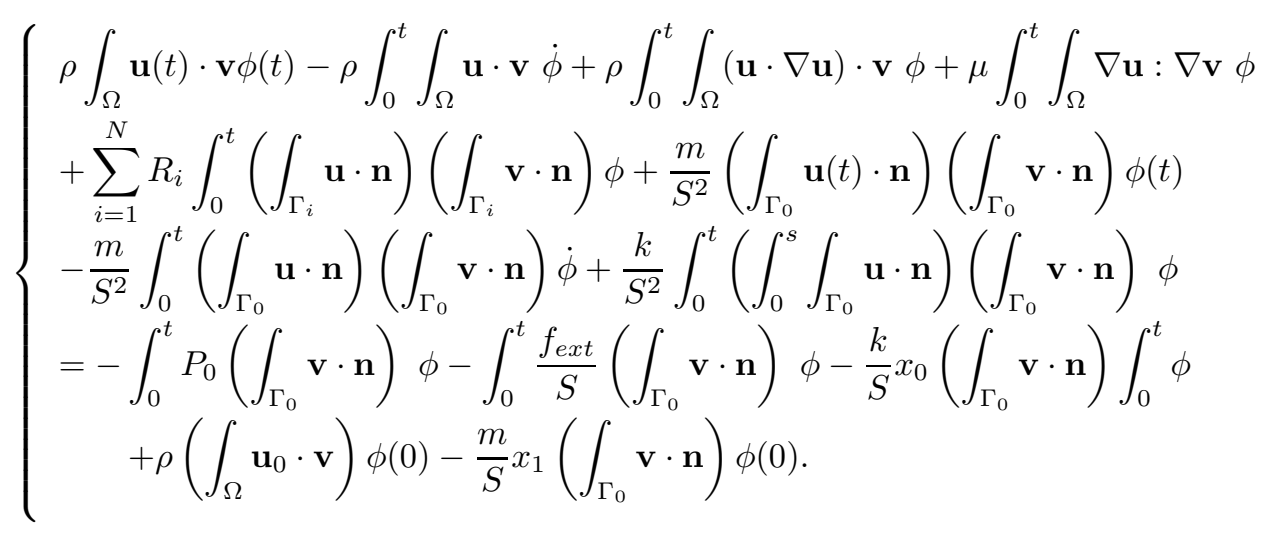

Note that here we have expressed all the quantities with the help of the fluid velocity. The velocity of the spring can be simply recovered by $S \dot{x}=-\int_{\Gamma_{0}} \mathbf{u} \cdot \mathbf{n}=-\lambda_{0} \int_{\Gamma_{0}} \mathbf{U}_{0} \cdot \mathbf{n}$.

\section{Existence Results}

One aim of the paper is to prove the existence of weak solutions as defined in the previous section. We have two types of existence results: one locally in time for large data, and one globally in time for small enough initial data in the special case where no external efforts are applied to the coupled system. Note moreover that in the case where the stifness of the spring is equal to zero, one could easily derive an existence result, globally in time, provided that the data (initial data and applied forces) are small enough. More precisely

Theorem 2.1. Under the assumptions (17) there exist a time $\tau_{0}, 0<\tau_{0}<T$ sufficiently small and a weak solution $\mathbf{u}$ of (18) on $\left[0, \tau_{0}\right]$. Moreover, there exists $C$ such that the solution satisfies the following estimate:

$$
\begin{gathered}
\rho\|\mathbf{u}\|_{L^{\infty}\left(0, \tau_{0} ; L^{2}(\Omega)\right)}^{2}+\frac{m}{S^{2}}\left\|\int_{\Gamma_{0}} \mathbf{u} \cdot \mathbf{n}\right\|_{L^{\infty}\left(0, \tau_{0}\right)}^{2}+\frac{k}{S^{2}}\left\|\int_{0}^{t} \int_{\Gamma_{0}} \mathbf{u} \cdot \mathbf{n}\right\|_{L^{\infty}\left(0, \tau_{0}\right)}^{2} \\
+\mu\|\mathbf{u}\|_{L^{2}\left(0, \tau_{0} ; H^{1}(\Omega)\right)}^{2}+\sum_{i=1}^{N} R_{i} \int_{0}^{\tau_{0}}\left(\int_{\Gamma_{i}} \mathbf{u} \cdot \mathbf{n}\right)^{2} \leq C .
\end{gathered}
$$

Furthermore, in the special case where the external efforts are equal to zero and if we assume that

$$
\frac{\rho}{2}\left\|\mathbf{u}_{0}\right\|_{L^{2}(\Omega)}^{2}+\frac{m}{2}\left|x_{1}\right|^{2}+\frac{k}{2}\left|x_{0}\right|^{2} \leq \frac{\mu^{2}}{8 \rho K^{2}}
$$


where $K$ has been defined in Section 1.1 (see (16)), then there exists at least one weak solution of (6) on [0, $\infty)$. Furthermore this solution satisfies:

$$
\frac{\rho}{2}\|\mathbf{u}\|_{L^{\infty}\left(0, \infty ; L^{2}(\Omega)\right.}^{2}+\frac{m}{2 S^{2}}\left\|\int_{\Gamma_{0}} \mathbf{u} \cdot \mathbf{n}\right\|_{L^{\infty}(0, \infty)}^{2}+\frac{k}{2}\left\|x_{0}+\frac{1}{S} \int_{0}^{t} \int_{\Gamma_{0}} \mathbf{u} \cdot \mathbf{n}\right\|_{L^{\infty}(0, \infty)}^{2} \leq \frac{\mu^{2}}{8 \rho K^{2}}
$$

and for any $T$

$$
\begin{gathered}
\rho\|\mathbf{u}\|_{L^{\infty}\left(0, T ; L^{2}(\Omega)\right)}^{2}+\frac{m}{S^{2}}\left\|\int_{\Gamma_{0}} \mathbf{u} \cdot \mathbf{n}\right\|_{L^{\infty}(0, T)}^{2}+\frac{k}{S^{2}}\left\|\int_{0}^{t} \int_{\Gamma_{0}} \mathbf{u} \cdot \mathbf{n}\right\|_{L^{\infty}(0, T)}^{2} \\
+\mu\|\mathbf{u}\|_{L^{2}\left(0, T ; H^{1}(\Omega)\right)}^{2}+\sum_{i=1}^{N} R_{i} \int_{0}^{T}\left(\int_{\Gamma_{i}} \mathbf{u} \cdot \mathbf{n}\right)^{2} \leq C_{T} .
\end{gathered}
$$

Remark 2.2. Note that in the special case where $k=0$ and if the data are small enough one can obtain an existence result globally in time.

\subsection{Proof of Theorem}

The proof of the existence results follows a standard procedure. In a first step we build a sequence of approximated solutions thanks to a Galerkin method. Then, since the energy bounds are not sufficient to pass to the limit in the weak formulations, we derive additional bounds. Applying a compactness result we then are able to pass to the limit in the system satisfied by the approximated solution leading to the existence of at least one weak solution.

\subsubsection{Galerkin Method}

The first step is to build a Galerkin basis of the space

$$
W=\left\{\mathbf{v} \in V, \exists \tilde{\Lambda}=\left(\tilde{\lambda}_{i}\right)_{0 \leq i \leq N} \text { s. t. } \mathbf{v}=\tilde{\lambda}_{i} \mathbf{U}_{i} \text { on } \Gamma_{i}, \quad i=0, \ldots, N\right\}
$$

Let $\mathbf{w}$ belong to $W$. We remark first that, taking into account the incompressibility constrain, it implies that $\sum_{i=0}^{N} \alpha_{i} \int_{\Gamma_{i}} \mathbf{U}_{i} \cdot \mathbf{n}=0$ with $\left.\mathbf{w}\right|_{\Gamma_{i}}=\alpha_{i} \mathbf{U}_{i}$. Consequently if $\int_{\Gamma_{i}} \mathbf{U}_{i} \cdot \mathbf{n}=0$ for all $0 \leq i \leq N$, the $\left(\alpha_{i}\right)$ belong to a vector space of dimension $N+1$ and if there exists $i_{0}$ such that $\int_{\Gamma i_{0}} \mathbf{U}_{i_{0}} \cdot \mathbf{n} \neq 0$ the $\left(\alpha_{i}\right)$ belong to a vectorial space of dimension $N$. Then, $\mathbf{w}=\mathbf{z}+\sum_{i \in J} \alpha_{i} \mathbf{w}_{i}$, where

$$
\mathbf{z} \in\left\{\mathbf{v} \in H_{0}^{1}(\Omega), \nabla \cdot \mathbf{v}=0\right\}
$$

and where $J$ and the $\mathbf{w}_{i} \in V$ are defined as follows:

$$
\text { - If } \int_{\Gamma_{i}} \mathbf{U}_{i} \cdot \mathbf{n}=0 \text { for all } 0 \leq i \leq N \text {, then } J=\{i, 0 \leq i \leq N\} \text { and }
$$

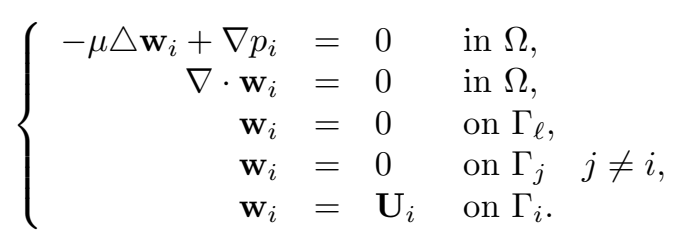

Note that this case is not likely to happen in the context of ventilation. 
- If there exists $i_{0}$ such that $\int_{\Gamma i_{0}} \mathbf{U}_{i_{0}} \cdot \mathbf{n} \neq 0$, then $J=\left\{i, 0 \leq i \leq N, i \neq i_{0}\right\}$ and we define the functions $\mathbf{w}_{i}, i \neq i_{0}$ as follows:

- for $i \neq i_{0}$

$$
\left\{\begin{aligned}
-\mu \Delta \mathbf{w}_{i}+\nabla p_{i} & =0 & & \text { in } \Omega, \\
\nabla \cdot \mathbf{w}_{i} & =0 & & \text { in } \Omega, \\
\mathbf{w}_{i} & =0 & & \text { on } \Gamma_{\ell}, \\
\mathbf{w}_{i} & =0 & & \text { on } \Gamma_{j} \quad j \neq i, i_{0}, \\
\mathbf{w}_{i} & =\beta_{i} \mathbf{U}_{i_{0}} & & \text { on } \Gamma_{i_{0}}, \\
\mathbf{w}_{i} & =\mathbf{U}_{i} & & \text { on } \Gamma_{i},
\end{aligned}\right.
$$

where $\beta_{i}=-\frac{\int_{\Gamma_{i}} \mathbf{U}_{i} \cdot \mathbf{n}}{\int_{\Gamma_{i_{0}}} \mathbf{U}_{i_{0}} \cdot \mathbf{n}}$.

Let $\left(\mathbf{z}_{n}\right)_{n \in \mathbb{N}}$ be a Galerkin basis of $\left\{\mathbf{v} \in H_{0}^{1}(\Omega), \nabla \cdot \mathbf{v}=0\right\}$. A Galerkin basis of $W$ is then $\left(\mathbf{z}_{n}\right)_{n \in \mathbb{N}} \cup\left(\mathbf{w}_{i}\right)_{0 \leq i \leq N}$. For all $m \in \mathbb{N}$, we define $\mathbf{u}_{m}(t) \in W_{m}=\operatorname{span}\left(\mathbf{z}_{n}\right)_{1 \leq n \leq m} \oplus \operatorname{span}\left(\mathbf{w}_{i}\right)_{1 \leq i \leq N}$ solution of the discrete problem $\left(P_{m}\right)$

$$
\left\{\begin{array}{l}
\rho \int_{\Omega} \frac{\partial \mathbf{u}_{m}}{\partial t} \cdot \mathbf{v}+\rho \int_{\Omega}\left(\mathbf{u}_{m} \cdot \nabla \mathbf{u}_{m}\right) \cdot \mathbf{v}+\mu \int_{\Omega} \nabla \mathbf{u}_{m}: \nabla \mathbf{v} \\
+\sum_{i=1}^{N} R_{i}\left(\int_{\Gamma_{i}} \mathbf{u}_{m} \cdot \mathbf{n}\right)\left(\int_{\Gamma_{i}} \mathbf{v} \cdot \mathbf{n}\right) \\
+\frac{m}{S^{2}} \frac{d}{d t}\left(\int_{\Gamma_{0}} \mathbf{u}_{m} \cdot \mathbf{n}\right)\left(\int_{\Gamma_{0}} \mathbf{v} \cdot \mathbf{n}\right)+\frac{k}{S^{2}} \int_{0}^{t}\left(\int_{\Gamma_{0}} \mathbf{u}_{m} \cdot \mathbf{n}\right)\left(\int_{\Gamma_{0}} \mathbf{v} \cdot \mathbf{n}\right) \\
=-P_{0}\left(\int_{\Gamma_{0}} \mathbf{v} \cdot \mathbf{n}\right)-\frac{f_{e x t}}{S} \int_{\Gamma_{0}} \mathbf{v} \cdot \mathbf{n}-\frac{k}{S^{2}} x_{0} \int_{\Gamma_{0}} \mathbf{v} \cdot \mathbf{n}, \quad \forall \mathbf{v} \in W_{m}, \\
\mathbf{u}_{m}(0)=\mathbf{u}_{0 m},
\end{array}\right.
$$

where $\mathbf{u}_{0 m} \in W_{m}$ and is such that $\mathbf{u}_{0 m}$ converges to $\mathbf{u}_{0}$ strongly in $H$ as $m$ goes to infinity.

Lemma 2.3. The discrete problem $\left(P_{m}\right)$ has a unique solution $\mathbf{u}_{m} \in W^{1, \infty}\left(0, T ; W_{m}\right)$. Moreover the following energy estimates are satisfied:

$$
\exists \tau_{0} \text { independent of } m, \text { s. } t .\left\|\mathbf{u}_{m}\right\|_{L^{\infty}\left(0, \tau_{0} ; L^{2}(\Omega)\right)}+\left\|\mathbf{u}_{m}\right\|_{L^{2}\left(0, \tau_{0} ; H^{1}(\Omega)\right)} \leq C
$$

where $C$ is a constant that does depends on $m$.

Furthermore, if the initial data satisfy (20), and if $P_{0}=0$ and $f_{\text {ext }}=0$ then

$$
\frac{\rho}{2}\left\|\mathbf{u}_{m}\right\|_{L^{\infty}\left(0, \infty ; L^{2}(\Omega)\right)}^{2}+\frac{m}{2 S^{2}}\left\|\int_{\Gamma_{0}} \mathbf{u}_{m} \cdot \mathbf{n}\right\|_{L^{\infty}(0, \infty)}^{2}+\frac{k}{2}\left\|x_{0}+\frac{1}{S} \int_{0}^{t} \int_{\Gamma_{0}} \mathbf{u}_{m} \cdot \mathbf{n}\right\|_{L^{\infty}(0, \infty)}^{2} \leq \frac{\mu^{2}}{8 \rho K^{2}}
$$

and for any $T$

$$
\begin{gathered}
\rho\left\|\mathbf{u}_{m}\right\|_{L^{\infty}\left(0, T ; L^{2}(\Omega)\right)}^{2}+\frac{m}{S^{2}}\left\|\int_{\Gamma_{0}} \mathbf{u}_{m} \cdot \mathbf{n}\right\|_{L^{\infty}(0, T)}^{2}+\frac{k}{S^{2}}\left\|\int_{0}^{t} \int_{\Gamma_{0}} \mathbf{u}_{m} \cdot \mathbf{n}\right\|_{L^{\infty}(0, T)}^{2} \\
+\mu\left\|\mathbf{u}_{m}\right\|_{L^{2}\left(0, T ; H^{1}(\Omega)\right)}^{2}+\sum_{i=1}^{N} R_{i} \int_{0}^{T}\left(\int_{\Gamma_{i}} \mathbf{u}_{m} \cdot \mathbf{n}\right)^{2} \leq C_{T} .
\end{gathered}
$$


Proof. It is clear that there exists a time $\tau_{m}$ such that the discrete problem $\left(P_{m}\right)$ has a unique solution

$$
\mathbf{u}_{m} \in W^{1, \infty}\left(0, \tau_{m} ; W_{m}\right)
$$

In particular the mass matrix defined by

$$
M_{i j}=\rho \int_{\Omega} \phi_{i} \cdot \phi_{j}+\frac{m}{S^{2}}\left(\int_{\Gamma_{0}} \phi_{i} \cdot \mathbf{n}\right)\left(\int_{\Gamma_{0}} \phi_{j} \cdot \mathbf{n}\right) \text { with } \phi_{i}, \phi_{j} \in W_{m}
$$

is invertible. Moreover this solution satisfies the estimates (13) derived at Section 1.1 and consequently $\tau_{m}=\tau_{0}$. Finally, by applying the same argument as the one we used at Section 1.1 we obtain the other desired bounds.

At least for a subsequence of $\mathbf{u}_{m}$ (still denoted by $\mathbf{u}_{m}$ ), these estimates yield the following weak convergences, as $m$ goes to $\infty$ :

$$
\left\{\begin{array}{l}
\mathbf{u}_{m} \rightarrow \mathbf{u} \text { weakly in } L^{2}(0, T ; V) \\
\mathbf{u}_{m} \rightarrow \mathbf{u} \text { weakly } \text { * in } L^{\infty}(0, T ; H),
\end{array}\right.
$$

where $T=\tau_{0}$ in the general case and $T$ can be any time in the case of small enough data. Moreover the limit $\mathbf{u}$ satisfies the same energy estimate as $\mathbf{u}_{m}$ thus inequality (19) is satisfied as well as (21), (22) in the case where no external forces are applied and (20) is verified. Nevertheless the previous convergences are not sufficient to pass to the limit in the weak formulation $\left(P_{m}\right)$ and in particular in the convection term. Consequently we need to derive additional bounds in order to obtain compactness of the sequence of approximated solution $\left(\mathbf{u}_{m}\right)_{m}$.

\subsubsection{Additional bounds}

We will follow exactly the same lines as in $[7,10]$. First we define

$$
H^{\gamma}\left(\mathbb{R} ; B_{0}, B_{1}\right)=\left\{\mathbf{v}, \mathbf{v} \in L^{2}\left(\mathbb{R}, B_{0}\right), D_{t}^{\gamma} \mathbf{v} \in L^{2}\left(\mathbb{R}, B_{1}\right)\right\}
$$

with $D_{t}^{\gamma} \mathbf{v}$ is the fractionnal derivative of order $\gamma$ in time of $\mathbf{v}$, defined as the inverse Fourier transform of $(2 i \pi \tau)^{\gamma} \hat{\mathbf{u}}(\tau)$. The space $H^{\gamma}\left(\mathbb{R} ; B_{0}, B_{1}\right)$ is endowed with the norm

$$
\|\mathbf{u}\|_{H^{\gamma}\left(\mathbb{R} ; B_{0}, B_{1}\right)}=\left(\|\mathbf{u}\|_{L^{2}\left(\mathbb{R} ; B_{0}\right)}^{2}+\left\||\tau|^{\gamma} \hat{\mathbf{u}}\right\|_{L^{2}\left(\mathbb{R} ; B_{1}\right)}^{2}\right)^{\frac{1}{2}}
$$

We define also $H^{\gamma}\left(0, T ; B_{0}, B_{1}\right)$, as the space of function obtained by restriction to $[0, T]$ of functions of $H^{\gamma}\left(\mathbb{R} ; B_{0}, B_{1}\right)$.

We will apply the following lemma that can be found also in [10]:

Lemma 2.4. Let $B_{0}, B$, and $B_{1}$ be three Hilbert spaces such that $B_{0} \subset B \subset B_{1}$ and such that $B_{0}$ is compactly embedded in $B$. Then for all $\gamma>0$, the injection $H^{\gamma}\left(0, T ; B_{0}, B_{1}\right) \rightarrow L^{2}(0, T ; B)$ is compact.

We are going to apply this lemma with

$$
B_{0}=V, B_{1}=H, \gamma=\frac{1}{4}-\varepsilon \text { and } B=H
$$

Indeed the additional estimate holds true:

Lemma 2.5. The sequence $\mathbf{u}_{m}$ is bounded in $H^{\gamma}(0, T ; V, H)$ for $0 \leq \gamma \leq \frac{1}{4}-\varepsilon$. 
Proof. We denote by $\overline{\mathbf{u}}_{m}$ the extention of $\mathbf{u}_{m}$ by zero 0 for $t<0$ and $t>T$, and $\hat{\mathbf{u}}_{m}$ the Fourier transform in time of $\overline{\mathbf{u}}_{m}$. Since we already know that $\mathbf{u}_{m}$ is uniformly bounded in $L^{2}(0, T, V)$, it remains to prove that

$$
\int_{-\infty}^{+\infty}|\tau|^{2 \gamma}\left\|\hat{\mathbf{u}}_{m}\right\|_{L^{2}(\Omega)}^{2} \leq C
$$

Then applying Lemma 2.4 we have that $\mathbf{u}_{m}$ is compact in $L^{2}(0, T ; H)$.

We have that $\overline{\mathbf{u}}_{m}$ satisties

$$
\begin{aligned}
& \rho \int_{\Omega} \frac{\partial \overline{\mathbf{u}}_{m}}{\partial t} \cdot \mathbf{v}+\rho \int_{\Omega}\left(\overline{\mathbf{u}}_{m} \cdot \nabla \overline{\mathbf{u}}_{m}\right) \cdot \mathbf{v}+\mu \int_{\Omega} \nabla \overline{\mathbf{u}}_{m}: \nabla \mathbf{v}+\sum_{i=1}^{N} R_{i}\left(\int_{\Gamma_{i}} \overline{\mathbf{u}}_{m} \cdot \mathbf{n}\right)\left(\int_{\Gamma_{i}} \mathbf{v} \cdot \mathbf{n}\right) \\
& +\frac{m}{S^{2}} \frac{d}{d t}\left(\int_{\Gamma_{0}} \overline{\mathbf{u}}_{m} \cdot \mathbf{n}\right)\left(\int_{\Gamma_{0}} \mathbf{v} \cdot \mathbf{n}\right)-\frac{k}{S} \bar{x}_{m}\left(\int_{\Gamma_{0}} \mathbf{v} \cdot \mathbf{n}\right) \\
& =-\bar{P}_{0}\left(\int_{\Gamma_{0}} \mathbf{v} \cdot \mathbf{n}\right)-\frac{\bar{f}_{e x t}}{S} \int_{\Gamma_{0}} \mathbf{v} \cdot \mathbf{n}-\rho \int_{\Omega} \overline{\mathbf{u}}_{m}(T) \cdot \mathbf{v} \delta_{T}+\rho \int_{\Omega} \overline{\mathbf{u}}_{m}(0) \cdot \mathbf{v} \delta_{0} \\
& -\frac{m}{S^{2}}\left(\int_{\Gamma_{0}} \mathbf{u}_{m}(T) \cdot \mathbf{n}\right)\left(\int_{\Gamma_{0}} \mathbf{v} \cdot \mathbf{n}\right) \delta_{T}+\frac{m}{S^{2}}\left(\int_{\Gamma_{0}} \mathbf{u}_{m 0} \cdot \mathbf{n}\right)\left(\int_{\Gamma_{0}} \mathbf{v} \cdot \mathbf{n}\right) \delta_{0} \quad \forall \mathbf{v} \in W_{m},
\end{aligned}
$$

where $x_{m}=x_{0}-\frac{1}{S} \int_{0}^{t} \int_{\Gamma_{0}} \mathbf{u}_{m} \cdot \mathbf{n}$. We now apply the Fourier transform to the previous equation and take $\hat{\mathbf{u}}_{m}$ as a test function, it yields

$$
\begin{aligned}
& \rho 2 i \pi \tau \int_{\Omega}\left|\hat{\mathbf{u}}_{m}(\tau)\right|^{2}+\rho \int_{\Omega} \hat{G}(\tau) \hat{\mathbf{u}}_{m}(\tau)+\mu \int_{\Omega} \nabla \hat{\mathbf{u}}_{m}(\tau): \nabla \hat{\mathbf{u}}_{m}(\tau)+\sum_{i=1}^{N} R_{i}\left(\int_{\Gamma_{i}} \hat{\mathbf{u}}_{m}(\tau) \cdot \mathbf{n}\right)^{2} \\
& +\frac{m}{S^{2}} 2 i \pi \tau\left(\int_{\Gamma_{0}} \hat{\mathbf{u}}_{m}(\tau) \cdot \mathbf{n}\right)^{2}+k 2 i \pi \tau\left(\hat{x}_{m}(\tau)\right)^{2}=-\left(\hat{P}_{0}(\tau)+\frac{\hat{f}_{e x t}}{S}(\tau)\right) \int_{\Gamma_{0}} \hat{\mathbf{u}}_{m}(\tau) \cdot \mathbf{n} \\
& -\rho \int_{\Omega} \overline{\mathbf{u}}_{m}(T) \cdot \hat{\mathbf{u}}_{m}(\tau) e^{-2 i \pi \tau T}+\rho \int_{\Omega} \overline{\mathbf{u}}_{m}(0) \cdot \hat{\mathbf{u}}_{m}(\tau) \\
& +\frac{m}{S^{2}}\left(\int_{\Gamma_{0}} \hat{\mathbf{u}}_{m}(\tau) \cdot \mathbf{n}\right)\left(\int_{\Gamma_{0}} \overline{\mathbf{u}}_{m}(T) \cdot \mathbf{n}\right) e^{-2 i \pi \tau T}-\frac{m}{S^{2}}\left(\int_{\Gamma_{0}} \hat{\mathbf{u}}_{m}(\tau) \cdot \mathbf{n}\right)\left(\int_{\Gamma_{0}} \overline{\mathbf{u}}_{m}(0) \cdot \mathbf{n}\right)
\end{aligned}
$$

where $\hat{G}$ is the Fourier transform with respect to the time of the nonlinear convection term $\overline{\mathbf{u}}_{m} \cdot \nabla \overline{\mathbf{u}}_{m}$.

Taking the imaginary part of the previous equality and using the inequality $\left|\int_{\Gamma_{0}} \mathbf{v} \cdot \mathbf{n}\right| \leq C\|\mathbf{v}\|_{L^{2}(\Omega)}$, for every $\mathbf{v} \in W$ (see Lemma 1.1), we have

$$
\begin{aligned}
& |\tau|\left\|\hat{\mathbf{u}}_{m}(\tau)\right\|_{L^{2}(\Omega)}^{2} \leq C\left\|\hat{G}_{m}(\tau)\right\|_{V^{\prime}}\left\|\hat{\mathbf{u}}_{m}(\tau)\right\|_{V}+C\left(\left\|\overline{\mathbf{u}}_{m}(T)\right\|_{L^{2}(\Omega)}+\left\|\overline{\mathbf{u}}_{m}(0)\right\|_{L^{2}(\Omega)}\right)\left\|\hat{\mathbf{u}}_{m}(\tau)\right\|_{L^{2}(\Omega)} \\
& +C\left|\hat{P}_{0}(\tau)+\frac{\hat{f}_{e x t}(\tau)}{S}\right|\left\|\hat{\mathbf{u}}_{m}(\tau)\right\|_{L^{2}(\Omega)}+C\left(\int_{\Gamma_{0}}\left|\overline{\mathbf{u}}_{m}(T) \cdot \mathbf{n}\right|+\int_{\Gamma_{0}}\left|\overline{\mathbf{u}}_{m}(0) \cdot \mathbf{n}\right|\right)\left\|\hat{\mathbf{u}}_{m}(\tau)\right\|_{L^{2}(\Omega)} .
\end{aligned}
$$

Moreover we have

$$
\left\|G_{m}\right\|_{V^{\prime}} \leq\left\|\mathbf{u}_{m}\right\|_{H^{1}(\Omega)}^{2}
$$

and then, thanks to the energy estimates satisfied by $\mathbf{u}_{m}$,

$$
\int_{-\infty}^{+\infty}\left\|G_{m}\right\|_{V^{\prime}} d t \leq C \int_{0}^{T}\left\|\mathbf{u}_{m}(t)\right\|_{H^{1}(\Omega)}^{2} d t \leq C
$$


from which we deduce

$$
\left\|\hat{G}_{m}(\tau)\right\|_{V^{\prime}} \leq C
$$

Futhermore, thanks to the energy estimates satisfied by $\mathbf{u}_{m}$ (see Lemma 2.3), we have that $\left\|\mathbf{u}_{m}(T)\right\|_{L^{2}(\Omega)} \leq C$ and since $\left\|\mathbf{u}_{m}(0)\right\|_{L^{2}(\Omega)} \leq C$, we obtain

$$
|\tau|\left\|\hat{\mathbf{u}}_{m}(\tau)\right\|_{L^{2}(\Omega)}^{2} \leq C\left(\left\|\hat{\mathbf{u}}_{m}(\tau)\right\|_{H^{1}(\Omega)}+\left\|\hat{\mathbf{u}}_{m}(\tau)\right\|_{L^{2}(\Omega)}\right) C\left\|\hat{\mathbf{u}}_{m}(\tau)\right\|_{H^{1}(\Omega)} .
$$

But, for $0<\gamma<\frac{1}{4}$, we see that

$$
|\tau|^{2 \gamma} \leq C(\gamma) \frac{1+|\tau|}{1+|\tau|^{1-2 \gamma}}, \forall \tau \in \mathbb{R}
$$

then

$$
\int_{-\infty}^{+\infty}|\tau|^{2 \gamma}\left\|\hat{\mathbf{u}}_{m}(\tau)\right\|_{L^{2}(\Omega)}^{2} \leq C(\gamma) \int_{-\infty}^{+\infty} \frac{\left\|\hat{\mathbf{u}}_{m}(\tau)\right\|_{H^{1}(\Omega)}}{1+|\tau|^{1-2 \gamma}}+C(\gamma) \int_{-\infty}^{+\infty} \frac{\left|\tau\left\|\mid \hat{\mathbf{u}}_{m}(\tau)\right\|_{H^{1}(\Omega)}\right.}{1+|\tau|^{1-2 \gamma}}
$$

The first integral of right hand side can be estimated

$$
\int_{-\infty}^{+\infty} \frac{\left\|\hat{\mathbf{u}}_{m}(\tau)\right\|_{H^{1}(\Omega)}^{2}}{1+|\tau|^{1-2 \gamma}} \leq\left(\int_{-\infty}^{+\infty} \frac{1}{\left(1+|\tau|^{1-2 \gamma}\right)^{2}}\right)^{\frac{1}{2}}\left(\int_{-\infty}^{+\infty}\left\|\hat{\mathbf{u}}_{m}(\tau)\right\|_{H^{1}(\Omega)}^{2}\right)^{\frac{1}{2}}
$$

The first integral is convergent for any $0<\gamma<\frac{1}{4}$. On the other hand, from Parseval equality

$$
\int_{-\infty}^{+\infty}\left\|\hat{\mathbf{u}}_{m}(\tau)\right\|_{H^{1}(\Omega)}^{2} d \tau=\int_{0}^{T}\left\|\mathbf{u}_{m}(t)\right\|_{H^{1}(\Omega)}^{2} d t \leq C
$$

With the same kind of arguments the second integral is convergent.

\subsubsection{Passage to the limit}

The previous compactness result imply the following strong convergence (at least for a subsequence of $\mathbf{u}_{m}$ still denoted $\mathbf{u}_{m}$ )

$$
\mathbf{u}_{m} \rightarrow \mathbf{u} \text { strongly in } L^{2}\left(0, T ; L^{2}(\Omega)\right) .
$$

This convergence result together with (24) enable us to pass to the limit in the following weak formulation (obtained from $\left(P_{m}\right)$ by multiplication by $\phi \in \mathcal{D}([0, T))$ and integration by parts in time)

$$
\left\{\begin{array}{l}
\rho \int_{\Omega} \mathbf{u}_{m}(t) \cdot \mathbf{v} \phi(t)-\rho \int_{0}^{t} \int_{\Omega} \mathbf{u}_{m} \cdot \mathbf{v} \dot{\phi}+\rho \int_{0}^{t} \int_{\Omega}\left(\mathbf{u}_{m} \cdot \nabla \mathbf{u}_{m}\right) \cdot \mathbf{v} \phi+\mu \int_{0}^{t} \int_{\Omega} \nabla \mathbf{u}_{m}: \nabla \mathbf{v} \phi \\
+\sum_{i=1}^{N} R_{i} \int_{0}^{t}\left(\int_{\Gamma_{i}} \mathbf{u}_{m} \cdot \mathbf{n}\right)\left(\int_{\Gamma_{i}} \mathbf{v} \cdot \mathbf{n}\right) \phi+\frac{m}{S^{2}}\left(\int_{\Gamma_{0}} \mathbf{u}_{m}(t) \cdot \mathbf{n}\right)\left(\int_{\Gamma_{0}} \mathbf{v} \cdot \mathbf{n}\right) \phi(t) \\
-\frac{m}{S^{2}} \int_{0}^{t}\left(\int_{\Gamma_{0}} \mathbf{u}_{m} \cdot \mathbf{n}\right)\left(\int_{\Gamma_{0}} \mathbf{v} \cdot \mathbf{n}\right) \dot{\phi}+\frac{k}{S^{2}} \int_{0}^{t}\left(\int_{0}^{s} \int_{\Gamma_{0}} \mathbf{u}_{m} \cdot \mathbf{n}\right)\left(\int_{\Gamma_{0}} \mathbf{v} \cdot \mathbf{n}\right) \phi \\
=-\int_{0}^{t} P_{0}\left(\int_{\Gamma_{0}} \mathbf{v} \cdot \mathbf{n}\right) \phi-\int_{0}^{t} \frac{f_{e x t}}{S}\left(\int_{\Gamma_{0}} \mathbf{v} \cdot \mathbf{n}\right) \phi-\frac{k}{S} x_{0}\left(\int_{\Gamma_{0}} \mathbf{v} \cdot \mathbf{n}\right) \int_{0}^{t} \phi \\
\quad-\rho\left(\int_{\Omega} \mathbf{u}_{m 0} \cdot \mathbf{v}\right) \phi(0)-\frac{m}{S} x_{1}^{m}\left(\int_{\Gamma_{0}} \mathbf{v} \cdot \mathbf{n}\right) \phi(0), \quad \forall \mathbf{v} \in W_{m},
\end{array}\right.
$$

where $x_{1}^{m}$ is equal to $-\frac{1}{S} \int_{\Gamma_{0}} \mathbf{u}_{0 m} \cdot \mathbf{n}$ and converges towards $x_{1}=-\frac{1}{S} \int_{\Gamma_{0}} \mathbf{u}_{0} \cdot \mathbf{n}$ as $m$ goes to infinity. Consequently we obtain the existence of at least one weak solution locally in time in the general case or globally in time for small enough data. 


\section{NumericAl illustrations}

In this section we present $2 D$ numerical results performed with Freefem $++($ see $[6])$. Our main purpose is to illustrate how this approach makes it possible to investigate the effect of a modification of some resistances in the condensed (distal) part onto the overall flow in the upper (proximal) part of the tree. The numerical approach shall be described and justified in a forthcoming paper. We simply present in the next section the principles it lies on.

\subsection{Numerical scheme}

We propose a time discretization of (6) based on the method of caracteristics for the convection part. Let $t^{0}=0<t^{1}<t^{2}<\cdots<t^{N}=T$, with $t^{n+1}-t^{n}=\delta t=T / N$, denote the time steps. We denote by $\left(\mathbf{u}^{n}, p^{n}\right)$ approximations of the velocity and pressure at time $t^{n}$, built recursively according to the following scheme :

$$
\begin{array}{rlrl}
\rho \frac{1}{\delta t} \mathbf{u}^{n+1}-\mu \Delta \mathbf{u}^{n+1}+\nabla p^{n+1} & =\rho \frac{1}{\delta t} \mathbf{u}^{n} \circ \mathbf{X}^{n} & & \text { in } \Omega \\
\nabla \cdot \mathbf{u}^{n+1} & =0 & & \text { in } \Omega \\
\mathbf{u}^{n+1} & =0 & & \text { in } \Gamma_{\ell} \\
\mathbf{u}^{n+1} & =\lambda_{i}^{n+1} \mathbf{U}_{i} & & \text { on } \Gamma_{i}, i=0 \ldots N \\
\int_{\Gamma_{0}}\left(\mu \nabla \mathbf{u}^{n+1} \cdot \mathbf{n}-p^{n+1} \mathbf{n}\right) \cdot \mathbf{U}_{0} & =-P_{0} \int_{\Gamma_{0}} \mathbf{U}_{0} \cdot \mathbf{n} & \\
\int_{\Gamma_{i}}\left(\mu \nabla \mathbf{u}^{n+1}-p^{n+1} \mathbf{n}\right) \cdot \mathbf{U}_{i} & =-P^{n+1} \int_{\Gamma_{i}} \mathbf{U}_{i} \cdot \mathbf{n}-R_{i} \lambda_{i}^{n+1}\left(\int_{\Gamma_{i}} \mathbf{U}_{i} \cdot \mathbf{n}\right)^{2} & \\
P^{n+1}=\frac{1}{S} & \left(m \frac{x^{n+1}-2 x^{n}+x^{n-1}}{\delta t^{2}}+k x^{n+1}-F_{e x t}^{n+1}\right) & \\
& & & \\
& &
\end{array}
$$

where $Q_{0}^{n+1}=\int_{\Gamma_{0}} \mathbf{u}^{n+1} \cdot \mathbf{n}$, and $X^{n}(x)$ stands for $Y^{n}\left(x, t^{n}\right)$, backward characteristic defined as

$$
\left\{\begin{aligned}
\frac{\partial Y}{\partial t}(x, t) & =\mathbf{u}^{n}(Y) \\
Y\left(x, t^{n+1}\right) & =x
\end{aligned}\right.
$$

We may now write the variational formulation for one time step: suppose that $x^{n}, \mathbf{u}^{n}$ are known, the couple $\left(\mathbf{u}^{n+1}, p^{n+1}\right) \in\left\{\mathbf{v} \in H^{1}(\Omega), \mathbf{v}=0\right.$ on $\Gamma_{\ell}, \exists \tilde{\Lambda}=\left(\tilde{\lambda}_{i}\right)_{0 \leq i \leq N}$ s.t. $\mathbf{v}=\tilde{\lambda}_{i} \mathbf{U}_{i}$ on $\left.\Gamma_{i}, i=0, \ldots, N\right\} \times L^{2}(\Omega)$ is computed as the solution to

$$
\begin{aligned}
a\left(\mathbf{u}^{n+1}, \mathbf{v}\right)+b\left(\mathbf{v}, p^{n+1}\right)= & l^{n+1}(\mathbf{v}) \forall \mathbf{v} \in H^{1}(\Omega), \mathbf{v}=0 \text { on } \Gamma_{\ell}, \\
& \exists \tilde{\Lambda}=\left(\tilde{\lambda}_{i}\right)_{0 \leq i \leq N} \text { s.t. } \mathbf{v}=\tilde{\lambda}_{i} \mathbf{U}_{i} \text { on } \Gamma_{i}, i=0, \ldots, N \\
b\left(\mathbf{u}^{n+1}, q\right)= & 0 \forall q \in L^{2}(\Omega)
\end{aligned}
$$




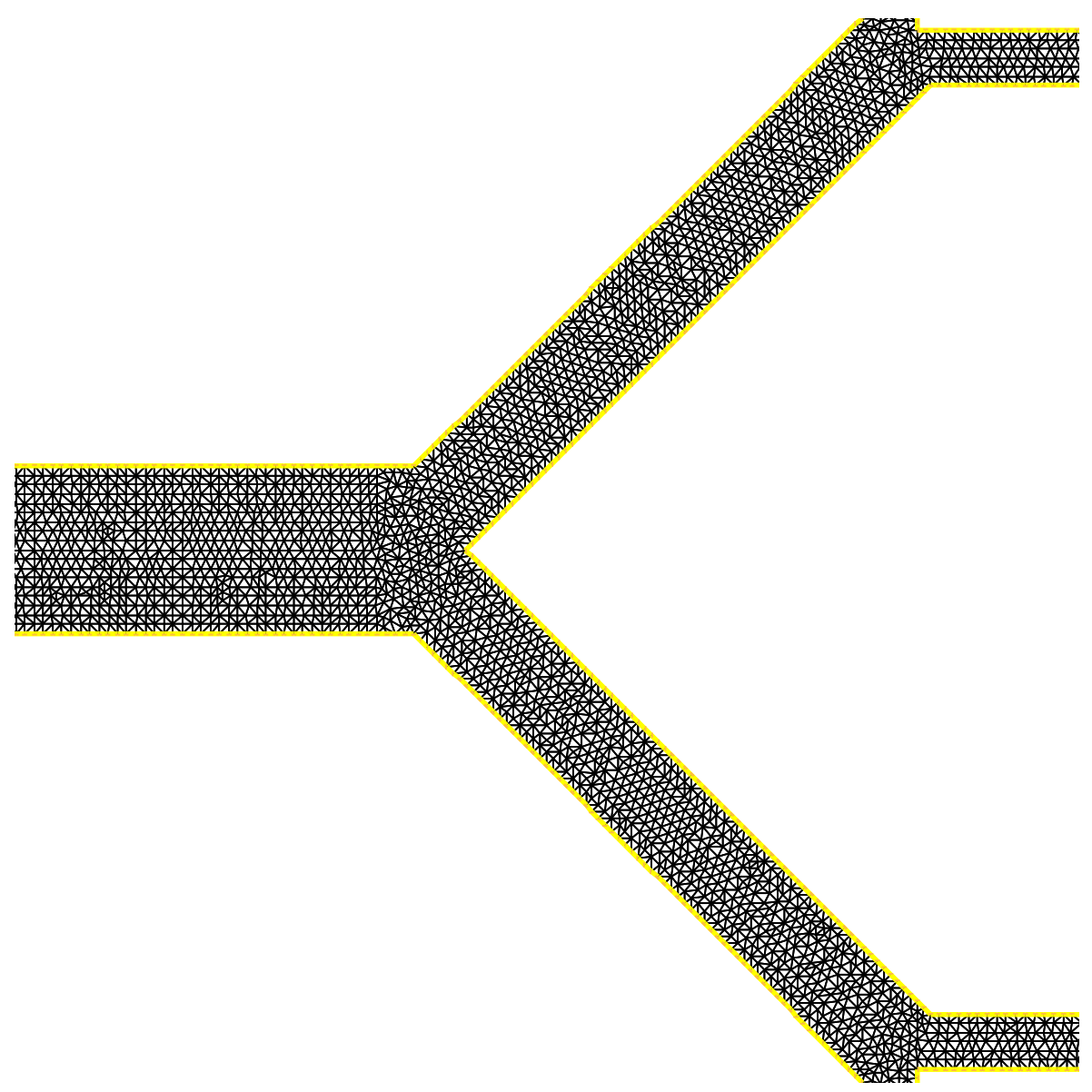

Figure 2. Mesh near the first bifurcation

where

$$
\begin{aligned}
& a\left(\mathbf{u}^{n+1}, \mathbf{v}\right)=\frac{\rho}{\delta t} \int_{\Omega} \mathbf{u}^{n+1} \cdot \mathbf{v}+\mu \int_{\Omega} \nabla \mathbf{u}^{n+1}: \nabla \mathbf{v} \\
&+\sum_{i=1}^{N} R_{i}\left(\int_{\Gamma_{i}} \mathbf{u}^{n+1} \cdot \mathbf{n}\right)\left(\int_{\Gamma_{i}} \mathbf{v} \cdot \mathbf{n}\right) \\
&+\frac{1}{S^{2}}\left(\frac{m}{\delta t}+k \delta t\right)\left(\sum_{i=1}^{N} \int_{\Gamma_{i}} \mathbf{u}^{n+1} \cdot \mathbf{n}\right)\left(\sum_{i=1}^{N} \int_{\Gamma_{i}} \mathbf{v} \cdot \mathbf{n}\right), \\
& l^{n+1}(\mathbf{v})=\frac{\rho}{\delta t} \int_{\Omega}\left(\mathbf{u}^{n} \circ \mathrm{X}^{n}\right) \cdot \mathbf{v}-P_{0} \int_{\Gamma_{0}} \mathbf{v} \cdot \mathbf{n}-\left(\frac{m}{\delta t S^{2}} Q_{0}^{n}+\frac{k}{S} x^{n}-\frac{f_{\text {ext }}^{n+1}}{S}\right)\left(\sum_{i=1}^{N} \int_{\Gamma_{i}} \mathbf{v} \cdot \mathbf{n}\right),
\end{aligned}
$$

and$$
b\left(\mathbf{u}^{n+1}, q\right)=-\int_{\Omega} \nabla \cdot \mathbf{u}^{n+1} q .
$$

Once $\mathbf{u}^{n+1}$ is known, $x^{n+1}$ is updated as

$$
x^{n+1}=x^{n}-\frac{\delta t}{S} Q_{0}^{n+1} \text { with } Q_{0}^{n+1}=\int_{\Gamma_{0}} \mathbf{u}^{n+1} \cdot \mathbf{n} .
$$




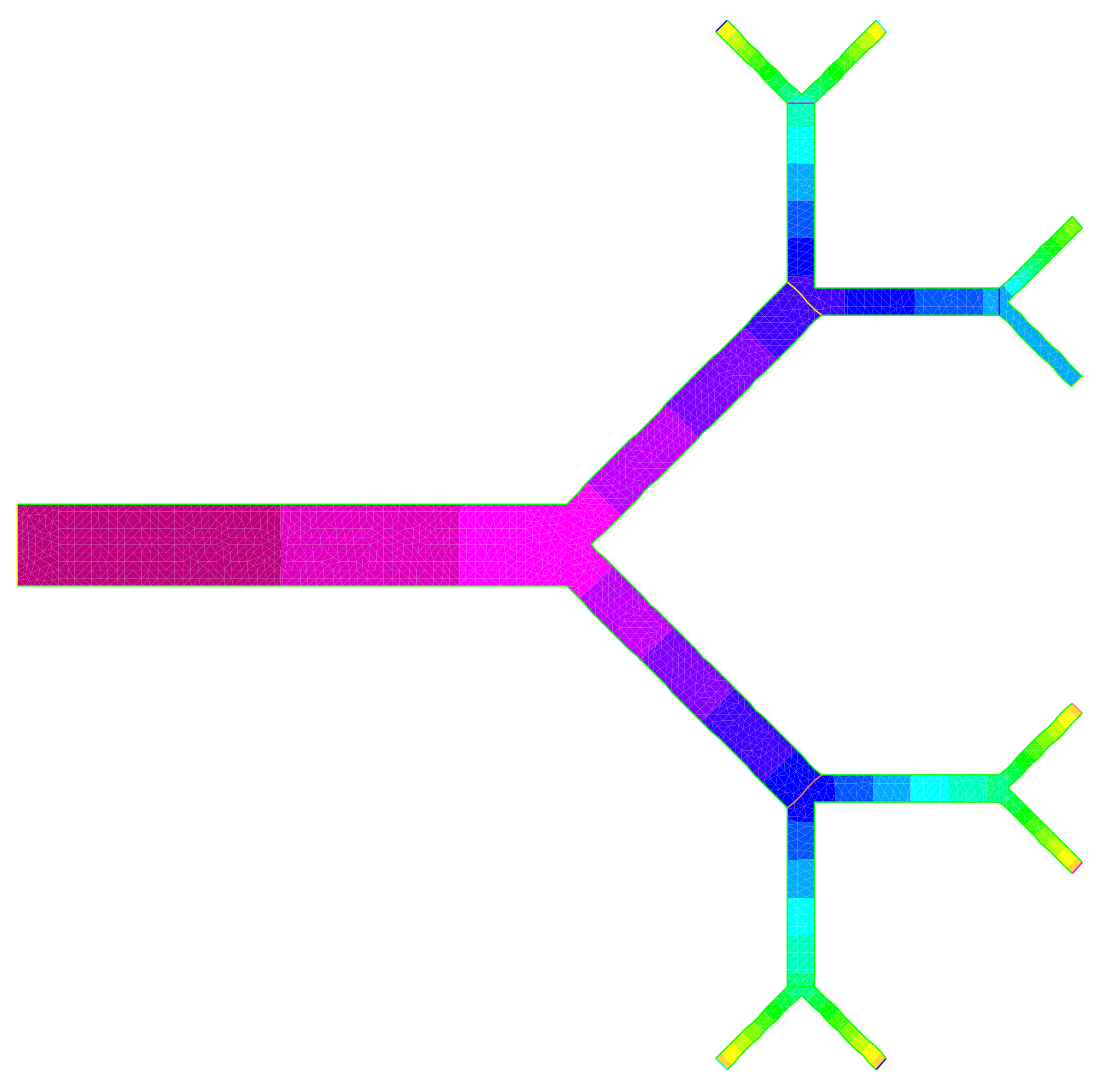

FiguRE 3. Pressure field in situation II

\subsection{Numerical tests}

To check the validity of this model, numerical tests were performed in [18], comparing a fully $2 \mathrm{D}$ model and a 2D-1D coupled model. The results obtained were in good agreement and the error was mainly due to a relatively bad geometrical approximation of the bifurcation area. Three dimensional computations shall be presented in a forthcoming paper. We simply present here some tests where Navier-Stokes simulations are performed on a $2 \mathrm{D}$ domain. From a modelling point of view, the suitability of this approach is highly questionable, as our $2 \mathrm{D}$ upper tree cannot be expected to reproduce all features of the real, three-dimensional one. Yet, we tried here to as respectful as possible of the mechanical properties of the human respiratory tree.

The main idea is to construct a two-dimensional tree equivalent to the three-dimensional one such that two conditions are fulfilled: the two trees have the same global resistance and the two-dimensional tree reproduces the resistance repartition of the three-dimensional tree. Consider the situation of geometrical trees, both 2D and $3 \mathrm{D}$, and introduce the ratios of homothety $h_{2 D}$ and $h_{3 D}$ respectively. Knowing that the $2 \mathrm{D}$ and $3 \mathrm{D}$ resistances are proportional to $\frac{1}{h_{2 D}^{2}}$ and $\frac{1}{h_{3 D}^{3}}$ respectively, we choose $h_{2 D}$ such that

$$
h_{2 D}=h_{3 D}^{\frac{3}{2}} .
$$




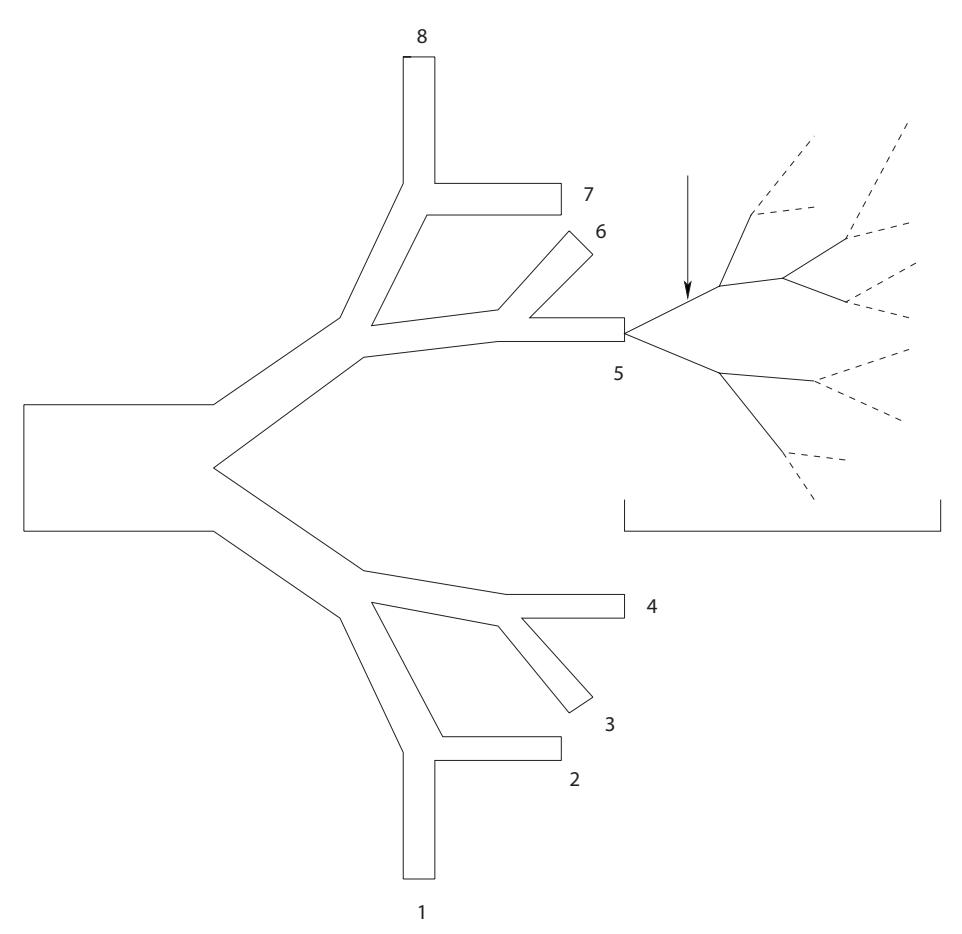

FIGURE 4. Resistance perturbation

For the generation zero, the trachea, we keep the real dimensions for the diameter and the length (width $=2 \cdot 10^{-2}$, lenght $\left.=12 \cdot 10^{-2}\right)$. As we know the global $3 \mathrm{D}$ resistance, we fix the fluid viscosity value such that both $2 \mathrm{~d}$ and $3 \mathrm{~d}$ global resistances are equal. Finally, the density is tuned according to the Reynolds number one wants to obtain (about 500 in the present case).

We consider the following set of data:

$$
m=0.4, S=0.011, k=2, \mu=410^{-3}, \rho=50 .
$$

All computations presented here are based on a non structured mesh, a part of which is represented in Fig 2. Initial conditions are $x=\dot{x}=0$, and the forcing term $f_{\text {ext }}$ is taken periodic (period of $5 s$ ), piecewise constant, with a 2 second active, inspiratory phase $\left(f_{\text {ext }}=0.1\right)$, followed by a passive, expiratory phase $\left(f_{\text {ext }}=0.0\right)$. Two situations are considered: in the first one (I), the 8 subtrees are identical, and each of them is symmetric (all resistances are the same in each generation). The second one is obtained as a perturbation of the first one: one of the resistances of subtree 5 (see the arrow on Fig. 4) is multiplied by $10^{4}$ (the corresponding is almost obstructed). Fig. 5 represents the corresponding $t \mapsto x(t)$ curves. As expected, the increase in resistance leads to a smaller ventilation amplitude. We plotted in Figure 6 the air fluxes through the 8 exits, at some time during the inspiration phase. The horizontal straight line corresponds to situation I: all fluxes are the same. The step function corresponds to situation II: one can check that the global flux is slightly reduced in the perturbed situation, but all fluxes are increased but through outlet 5, where it is reduced drastically. Note that the perturbation affects significantly outlet 6 , and much less outlet 4 . It is due to the fact that 5 and 6 are quite closed as ends of the tree, whereas 5 and 4 are not. 


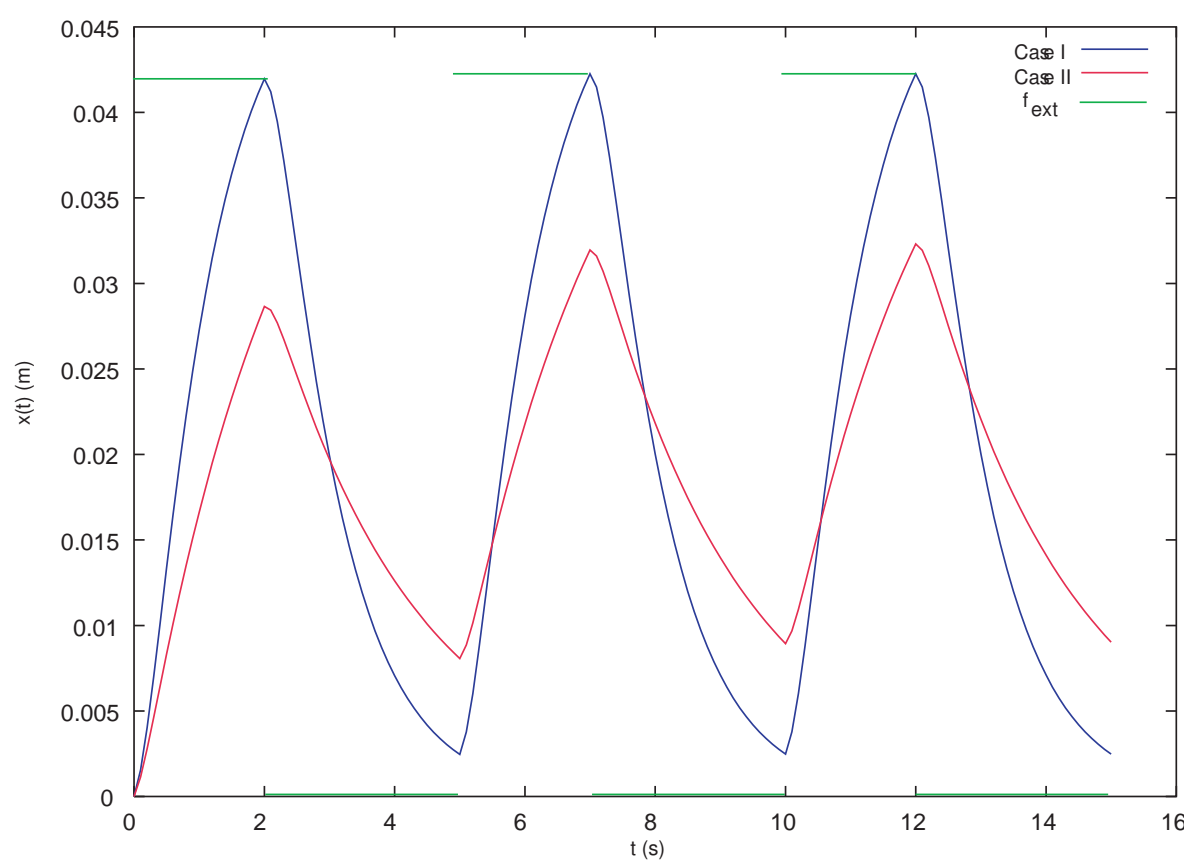

FigURE 5. Simulation of three respiratory cycles, cases I and II

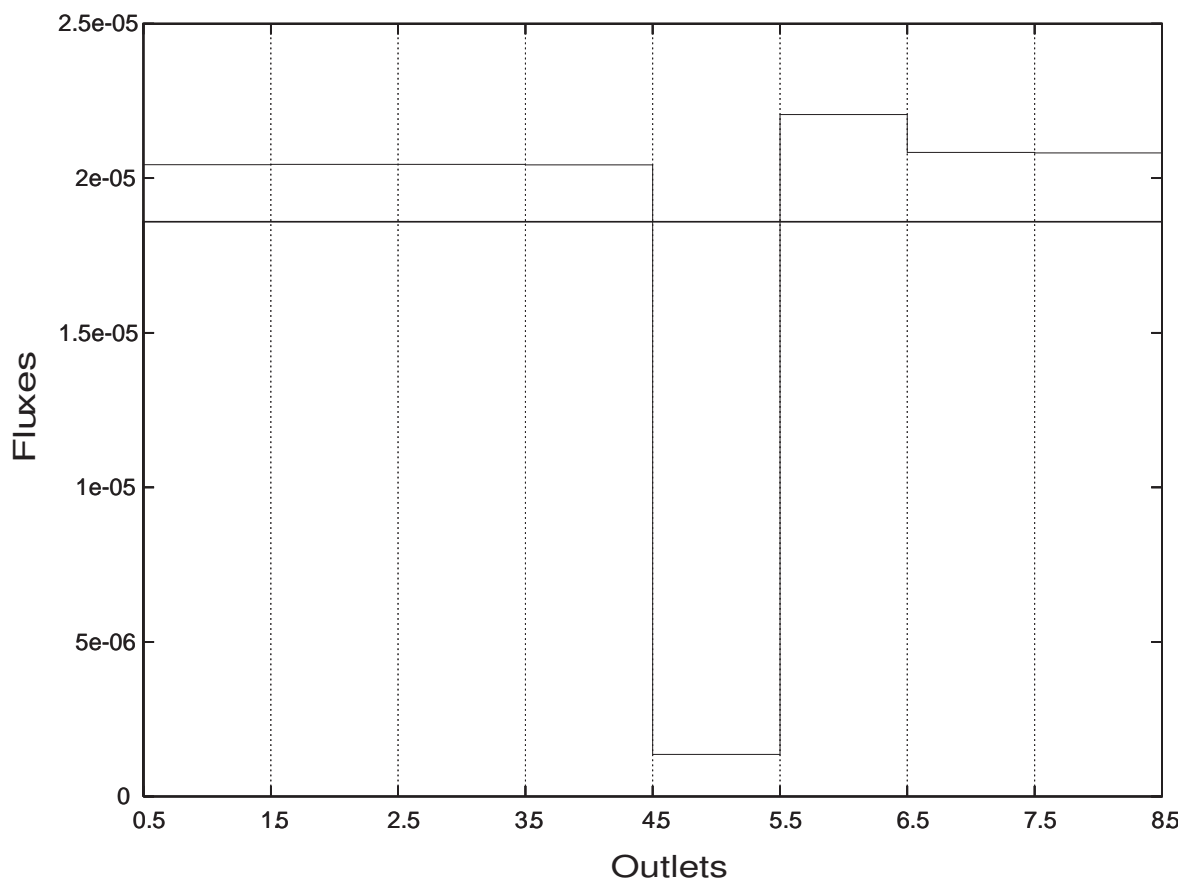

FiguRE 6. Fluxes through the outlets

\section{Concluding Remarks}

This article presents a general strategy to decompose the ventilation process onto interacting submodels, in order to restrict the expensive computations (resolution of Navier-Stokes equations) to the zone where they 
actually make sense, without sacrifying the overall suitability of the approach in terms of modelling. The obtained numerical results suggest a good behavior of the approach. In particular, some numerical comparisons between this 2D-0D model and the fully 2D model (the subtrees are not condensed) were performed in academic test cases, and they make clear that the approach is justified as soon as the Reynolds number in the condensed part is low. But the fact that only 2D Navier-Stokes equations are considered prevents us from making full comparisons with actual measurements. The real confrontation to reality, based on 3D computations, will be given in a forthcoming paper.

\section{REFERENCES}

[1] V. Antonaglia, L. Torelli, W.A. Zin and A. Gullo, Effects of viscoelasticity on volume distribution in a two-comportmental model of normal and sick lungs, Physiol. Meas. 26 (2005), 13-28.

[2] R. Begin, A.D. Renzetti Jr., A.H. Bigler and S. Watanabe, Flow and age dependence of airway closure and dynamic compliance, J. Appl. Physiol. 38 (1975), no 2, 199-207.

[3] W. Benish, P. Harper, J. Ward and J. Popovich, Jr., A mathematical model of lung static pressure-volume relationships: comparison of clinically derived parameters of elasticity, Henry Ford Hosp. Med. J. 36 (1988), no 1, 44-47.

[4] G.P.S. Crooke, J.D. Head and J.J. Marini, A general two-compartment model for mechanical ventilation, Math. Comput. Modelling 24 (1996), no. 7, 1-18.

[5] Y.H. Chang and C.P. Yu, A model of ventilation distribution in the human lung, Aer. Sci. Tech. 30 (1999), 309-319.

[6] http://www.freefem.org

[7] V. Girault, P.-A. Raviart, Finite Element Approximation of the Navier-Stokes Equations, Lecture Notes in Mathematics, Vol 749 Springer Verlag, Berlin, 1979.

[8] C. Grandmont, Y. Maday and B. Maury, A multiscale / multimodel approach of the respiration tree, Proceedings of the International Conference "New Trends in Continuum Mechanics" 8-12 September 2003, Constantza, Romania Theta Foundation Publications, Bucharest, 2005.

[9] C. Grandmont, B. Maury and N. Meunier, A viscoelastic model with non-local damping application to the human lungs, Math. Mod. Numer. Anal. 40 (2006), no 1, 201-224.

[10] J. L. Lions, Quelques méthodes de résolution des problèmes aux limites non linéaires, Dunod, 2002.

[11] S. Martin, B. Maury, T. Similowski, C. Straus, Modelling of respiratory system mechanics involving gas exchange in the human lungs, submitted to ESAIM PROC.

[12] B. Maury, N. Meunier, A. Soualah, L. Vial, Outlet Dissipative conditions for air flow in the bronchial tree, ESAIM Proceedings, september 2005, vol. 14, 115-123, Eric Cancès \& Jean-Frédéric Gerbeau, Editors.

[13] B. Mauroy, M. Filoche, J.S. Andrade Jr. and B. Sapoval, Interplay between flow distribution and geometry in an airway tree, Phys. Rev. Lett. 90, 14 (2003).

[14] Mathematical models in human physiology, J.T. Ottesen, M.S. Olufsen, J.K. Larsen (editors), SIAM, Philadelphia, 2004.

[15] A. Quarteroni, A. Veneziani, Analysis of a Geometrical Multiscale Model Based on the Coupling of ODEs and PDEs for Blood Flow Simulations, Multiscale Model. Simul., Vol 1, No. 2, 173-195, 2003.

[16] J.R. Rodarte and K. Rehder, Dynamic of respiration, In: Handbook of physiology: the respiratory system, A.P. Fishman, P.T. Macklem, J. Mead and S.R. Geiger (editors), Americal Physiological Society, 131-144, 1986.

[17] T. Similowski and J.H.T. Bates, Two-compartment modelling of respiratory system mechanics at low frequencies: gas redistribution or tissue rheology?, Eur. Respir. J. 4 (1991), 353-358.

[18] A. Soualah, Mathematical and numerical modelling of the humain lung, Doctoral Thesis, 2007.

[19] I. E. Vignon-Clementel, C. A. Figueroa, K. E. Jansen, C. A. Taylor, Outflow boundary conditions for three-dimensional finite element modelling of blood and pressure in arteries, Comput. Methods Appl. Mech. Engrg., 195, 3776-3796 , 2006.

[20] J.G. Venegas, R.S. Harris and B.A. Simon, A comprehensive equation for the pulmonary pressure-volume curve, J. Appl. Physiol. 81 (1998), no 1, 389-395.

[21] E.R. Weibel, Morphometry of the human lung, Springer Verlag and Academic Press, Berlin, New York, 151 pp., 1963.

[22] J.B. West, J.B. Respiratory Physiology - The Essentials, Baltimore: Williams \& Wilkins, 1974.

[23] The lung: scientific foundations, R.G. Crystal, J.B. West, E.R. Weibel and P.J. Barnes (editors), 2 Vol., 2nd Edition, Lippincott-Raven Press, Philadelphia, 1997. 